\title{
The effective temperature scale of giant stars (F0-K5)
}

\section{The effective temperature determination by means of the IRFM}

\author{
A. Alonso, S. Arribas, and C. Martínez-Roger \\ Instituto de Astrofísica de Canarias, E-38200 La Laguna, Tenerife, Spain \\ e-mail: aas@ll.iac.es, sam@ll.iac.es and cmr@ll.iac.es
}

Received December 28, 1998; accepted July 5, 1999

\begin{abstract}
We have applied the InfraRed Flux Method (IRFM) to a sample of approximately 500 giant stars in order to derive their effective temperatures with an internal mean accuracy of about $1.5 \%$ and a maximum uncertainty in the zero point of the order of $0.9 \%$. For the application of the IRFM, we have used a homogeneous grid of theoretical model atmosphere flux distributions developed by Kurucz (1993). The atmospheric parameters of the stars roughly cover the ranges: $3500 \mathrm{~K} \leq T_{\text {eff }} \leq 8000 \mathrm{~K} ;-3.0$ $\leq[\mathrm{Fe} / \mathrm{H}] \leq+0.5 ; 0.5 \leq \log (g) \leq 3.5$. The monochromatic infrared fluxes at the continuum are based on recent photometry with errors that satisfy the accuracy requirements of the work. We have derived the bolometric correction of giant stars by using a new calibration which takes the effect of metallicity into account. Direct spectroscopic determinations of metallicity have been adopted where available, although estimates based on photometric calibrations have been considered for some stars lacking spectroscopic ones. The adopted infrared absolute flux calibration, based on direct optical measurements of stellar angular diameters, puts the effective temperatures determined in this work in the same scale as those obtained by direct methods.

We have derived up to four temperatures, $T_{J}, T_{H}, T_{K}$ and $T_{L^{\prime}}$, for each star using the monochromatic fluxes at different infrared wavelengths in the photometric bands $J$, $H, K$ and $L^{\prime}$. They show good consistency over $4000 \mathrm{~K}$, and there is no appreciable trend with wavelength, metallicity and/or temperature.

We provide a detailed description of the steps followed for the application of the IRFM, as well as the sources of error and their effect on final temperatures. We also provide a comparison of the results with previous work.
\end{abstract}

Key words: stars: fundamental parameters - stars: Population II — stars: atmospheres - stars: general

$\overline{\text { Send offprint requests to: }}$ A. Alonso

\section{Introduction}

The scale of temperatures of giant stars has been the target of a number of previous studies based on both direct and indirect methods (e.g. Ridgway et al. 1980; Bell \& Gustafsson 1989; Blackwell et al. 1990; Mozurkewich et al. 1991; Richichi et al. 1992; Di Benedetto 1993, 1998; Dyck et al. 1998). In spite of these substantial efforts, very little attention has been paid to the role played by metallicity, especially in the case of direct methods given the restrictions on their application. Nevertheless, metallicity may have a non-negligible influence when applying the effective temperature scale to the analysis of important problems in astrophysics, such as the determination of chemical abundances from spectroscopy, the colour synthesis of stellar populations, the correct interpretation of the observed HR diagram and the observational test of stellar fluxes generated with atmospheric models.

The direct methods for measuring stellar angular diameters (i.e. mainly Michelson interferometry at different wavelengths and lunar occultation measurements) establish the empirical effective temperature scale of Population I giants $([\mathrm{Fe} / \mathrm{H}] \sim 0)$ from G0III to M8III. Even in this case, on the observational side the error sources affecting the processes of measurement and reduction of the data make it difficult to ascertain such basic questions as whether spherical effects in the extended atmopsheres of the cooler stars are relevant. On the theoretical side the application of the limb-darkening correction remains unsure. This entails uncertainties concerning the nature of the stellar atmospheres in the range of late spectral types and low surface gravities. The present status for metal-poor giant stars is still more uncertain, given that no interferometric measurements of stellar diameters are available over the whole range of temperatures. A number of indirect methods based on stellar atmosphere models may be applied to determine 
the effective temperatures of giant $\operatorname{stars}^{1}$ (see for instance the review by Böhm-Vitense 1981). Unfortunately, theoretically based temperatures are not as trustworthy as would be desirable since atmosphere models cannot reproduce the observed fluxes with the required accuracy, especially in the UV range (e.g. Morossi et al. 1993). The suspicion that these problems are related to the adopted metal line opacities - a probable excess opacity for giants (Malagnini et al. 1992)-makes the temperature scale of metal-deficient stars more uncertain. In addition to their partial dependence on models, most of the previous works concerning the $T_{\text {eff }}$ scale for metal-deficient giants have the additional drawback of the low number of stars. As a consequence, the uncertainties in the calibration of $T_{\text {eff }}$ versus colour and metallicity are larger than desirable.

In order to overcome the above mentioned disadvantages, we have carried out a programme aimed at a more reliable definition of the effective temperature scale of giant stars (F0-K5). This work is part of a long term programme aimed at a complete revision of the $T_{\text {eff }}$ scale of the different regions of the HR diagram. The work is based on the Infrared Flux Method (Blackwell et al. 1990), which has proven useful for deriving temperatures of metal-poor giants of globular clusters (Arribas \& Martínez-Roger 1987; Arribas et al. 1991), and has low dependence on models for these types of stars. The temperatures obtained are scaled to direct $T_{\text {eff }}$ (Alonso et al. 1994a; Paper II). A thorough account of the procedure followed for the application of the method can be found in (Alonso et al. 1996a; Paper I) where we described a similar programme devoted to main sequence stars.

As an initial step, we selected a sample of stars $(\sim 500)$ covering a wide range in metal content $(+0.5>[\mathrm{Fe} / \mathrm{H}]>$ -3.0 ), and measured the infrared photometry $J H K\left(L^{\prime}\right)$ required for the application of the IRFM (Alonso et al. 1998; Paper IV). The number of stars and their distribution in the parameters space is adequate for establishing reliable relations $T_{\text {eff }}-$ colour $-[\mathrm{Fe} / \mathrm{H}]$ for giant stars. In this paper, we present the temperatures obtained. In a forthcoming paper, we will provide and discuss the calibrations $T_{\text {eff }}-$ colour- $[\mathrm{Fe} / \mathrm{H}]$, as well as the mean intrinsic colours for giant stars.

The present paper is laid out as follows. In Sects. 2, 3 and 4 , we outline the practical implementation of the IRFM: i.e. the calibration of $q \times R$-factors by using the grid of atmosphere models computed by Kurucz (1991, 1993); The determination and calibration of bolometric fluxes of giant stars by applying a method previously devised to obtain and calibrate bolometric fluxes of main

\footnotetext{
${ }^{1}$ It is worth noting that, as implicitly mentioned, direct temperatures are not totally independent from models, since their determination demands limb-darkening corrections and the estimation of a part of the UV flux contributing to the bolometric flux.
}

sequence stars (Alonso et al. 1995; Paper III) and the description of the assignment of secondary atmospheric parameters to the stars of the sample. The effective temperatures are derived in Sect. 5, where we provide an analysis of the the internal consistency of the method and the uncertainties affecting the derived efective temperatures. In Sect. 6, temperatures derived in the present work are compared with those derived in previous works. In Sect. 7, results are summarized.

\section{The implementation of the IRFM}

In principle, stellar effective temperatures can be determined in a fundamental way by measuring both the angular diameter and the bolometric flux of stars. In practice, this direct approach is limited to very bright stars confined to the close solar neighbourhood. If, as in the present programme, we are interested in extending the temperature determination to other target stars, then we are compelled to use indirect methods. Among the latter, the InfraRed Flux Method (Blackwell et al. 1990) has proven its reliability and non-critical dependence on models for spectral types earlier than late $\mathrm{K}$ and/or M. A detailed description of the implementation of the IRFM adopted in the present work is given in Paper I. For the sake of self-consistency we provide below a brief outline of it.

The determination of $T_{\text {eff }}$ is obtained by comparing the quotient between the bolometric flux $\left(F_{\mathrm{Bol}}\right)$ and the monochromatic flux at a chosen infrared wavelength of the continuum $\left(F\left(\lambda_{\mathrm{IR}}\right)\right)$ both measured at the top of the Earth's atmosphere $\left(R_{\mathrm{obs}}\right)$, with the outputs of models $\left(R_{\text {theo }}\right)$. Therefore, the basic equation of the IRFM is:

$$
\begin{aligned}
R_{\mathrm{obs}}=\frac{F_{\mathrm{Bol}}}{F\left(\lambda_{\mathrm{IR}}\right)}= & \frac{\sigma T_{\mathrm{eff}}^{4}}{F_{\mathrm{mod}}\left(\lambda_{\mathrm{IR}}, T_{\mathrm{eff}},[\mathrm{Fe} / \mathrm{H}], g\right)}= \\
& =R_{\mathrm{theo}}\left(\lambda_{\mathrm{IR}}, T_{\mathrm{eff}},[\mathrm{Fe} / \mathrm{H}], g\right),
\end{aligned}
$$

where the dependence of models on metallicity, surface gravity, and $\lambda_{\mathrm{IR}}$ is explicitly taken into account. The monochromatic fluxes are obtained by applying the relation

$F\left(\lambda_{\mathrm{IR}}\right)=q\left(\lambda_{\mathrm{IR}}, T_{\mathrm{eff}},[\mathrm{Fe} / \mathrm{H}], g\right)\left[F_{\mathrm{cal}}\left(\lambda_{\mathrm{IR}}\right) 10^{-0.4\left(m-m_{\text {cal }}\right)}\right]$,

where $m$ and $m_{\text {cal }}$ are, respectively, the magnitudes of the problem and standard star, $\lambda_{\mathrm{IR}}$ is the selected wavelength at the infrared, $F_{\text {cal }}\left(\lambda_{\mathrm{IR}}\right)$ is the absolute flux of the standard star at $\lambda_{\mathrm{IR}}$, and $q\left(\lambda_{\mathrm{IR}}, T_{\mathrm{eff}},[\mathrm{Fe} / \mathrm{H}], g\right)$ is a dimensionless factor which corrects the effect of the different curvature of the flux density distribution, across the filter window, between the standard and the problem stars.

The separation of observational and model inputs is easily obtained by substituting relation (2) in Eq. (1) as follows:

$$
\begin{gathered}
\frac{F_{\mathrm{Bol}}}{F_{\mathrm{cal}}\left(\lambda_{\mathrm{IR}}\right) 10^{-0.4\left(m-m_{\mathrm{cal}}\right)}}= \\
=q\left(\lambda_{\mathrm{IR}}, T_{\mathrm{eff}},[\mathrm{Fe} / \mathrm{H}], g\right) R_{\mathrm{theo}}\left(\lambda_{\mathrm{IR}}, T_{\mathrm{eff}},[\mathrm{Fe} / \mathrm{H}], g\right) .
\end{gathered}
$$




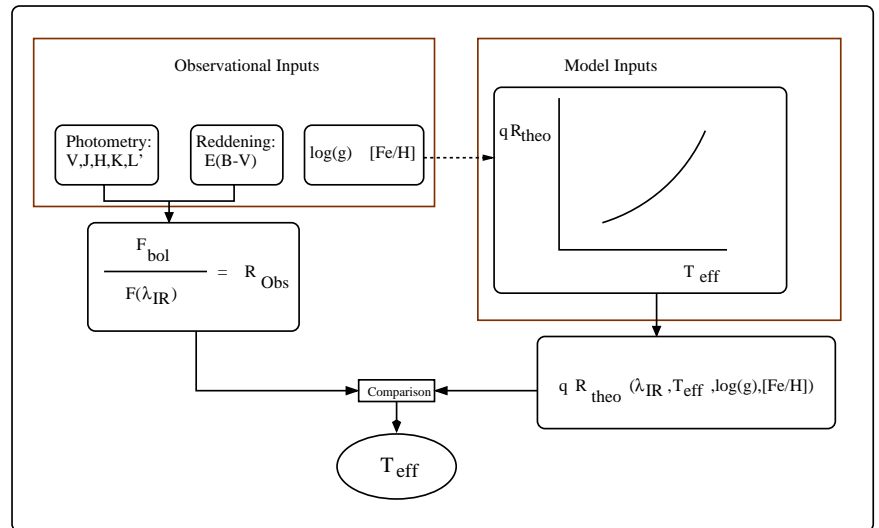

Fig. 1. Outline of the IRFM as implemented in the present work

Once $[\mathrm{Fe} / \mathrm{H}]$ and $\log (g)$ are known for a certain star, the observational quantities on the left-hand side of Eq. (3) determine the star's effective temperature by comparing with the theoretical values obtained from models on the right-hand side. An outline of the practical application of the IRFM is shown in Fig. 1.

\section{Model inputs: the $q-$ and $R$-factors}

The use of broad-band photometry to obtain the IR monochromatic fluxes requires the application of the socalled $q$-factors introduced in Eq. (2) (See also Paper I). Ideally, $q$-factors should be determined from spectroscopic data, but in the absence of a complete data base of empirical IR spectra, we have to rely on a grid of models to compute $q$-factors. Note however that $q$-factors always imply small corrections in the range studied.

The theoretical flux density distributions from Kurucz's $(1991,1993)$ models have been used to calculate $R_{\text {theo }}\left(\lambda_{\mathrm{IR}}\right)$ and $q\left(\lambda_{\mathrm{IR}}\right)$ factors, as defined in Eq. (1) and Eq. (2) respectively. The effective wavelengths of the bands $J, H, K$ and $L^{\prime}$ for the application of the IRFM were computed by considering the instrumental response of the photometric system (Alonso et al. 1994b and Paper IV) and the atmospheric transparency of the site computed by using the PLEXUS code (Clark 1996). Then the closest wavelengths sampled by the models were adopted $\left(\lambda_{J}=1272.5 \mathrm{~nm}, \lambda_{H}=1635.0 \mathrm{~nm}\right.$, $\lambda_{K}=2175.0 \mathrm{~nm}$ and $\left.\lambda_{L^{\prime}}=3690.0 \mathrm{~nm}\right)$.

Tables $1,2,3$ and 4 contain the calibration of $q$ and $R$-factors generated with Kurucz's models as a function of temperature, metallicity and surface gravity. Effective temperatures cover the range 3500-6500 K, surface gravities cover the range $\log (g)=(0.0-3.5)$, and metallicities cover the range $(0.5,-3.5)$.

\subsection{Sensitivity of $q \times R$ to the effective temperature}

The separation of terms in Eq. (3) (i.e. model information to the right-handside, and observational data to the lefthandside) is useful to simplify the analysis of the influence of errors on the derived temperatures.

Among the four near-IR wavelengths considered in this work, the $q \times R_{J}$ factors are the least sensitive to temperature, especially under $5000 \mathrm{~K}$. The $q \times R_{H}$ and $q \times R_{K}$ sensitivities are comparable, although temperatures lower than $4000 \mathrm{~K}$ derived using $q \times R_{H}$ are less reliable, due to the relative maximum of the flux associated with the minimum of the $\mathrm{H}^{-}$opacity reached in this band. As a consequence, the value of $q_{H}$ factors in this temperature range is significantly different from 1 , so that the effect of any possible uncertainty in the $\mathrm{H}^{-}$opacity or in other sources of opacity which now become more important is amplified. Finally, the highest sensitivity to $T_{\text {eff }}$ is shown by $q \times R_{L^{\prime}}$, however this sensitivity is counterbalanced by the difficult of measurement of fluxes in this band.

The variations induced by the change in metallicity or surface gravity are only important for $T_{\text {eff }}$ lower than $4250 \mathrm{~K}$. In particular, the variation of $R$-factors in the range $\log (g)=0-3.5$ for a fixed temperature and metallicity is almost negligible.

The values of $q \times R$ obtained from Tables $1-4$ allow the errors induced by the uncertainties in the different input parameters of the IRFM on the derived temperatures to be derived easily (Fig. 2).

If we consider a variation of $5 \%$ in $q \times R$ - the theoretical counterpart to the quotient $F_{\mathrm{Bol}} / F\left(\lambda_{\mathrm{IR}}\right)$ - the change in temperatures derived using the factors $R_{H}, R_{K}$ and $R_{L^{\prime}}$ is practically constant over $4000 \mathrm{~K}: 1.6-2 \%$ for $T_{H}$ and $1.6 \%$ for $T_{K}$ and $T_{L^{\prime}}$. The change of $T_{J}$ varies from $8 \%$ at $4000 \mathrm{~K}$ to $2 \%$ at $7000 \mathrm{~K}$. Hence, $R_{J}$ is the poorest indicator of $T_{\text {eff }}$ for the application of the IRFM, and we will consider only $T_{J}$ temperatures over $5000 \mathrm{~K}$ in the average. An uncertainty of 0.5 dex in metallicity causes, over $4000 \mathrm{~K}$, a maximum average error of $0.5 \%$ in the mean temperatures derived applying the IRFM (Fig. 2). The influence of an error of 0.5 dex in $\log (g)$ is even smaller on the derived temperatures, reaching at most average errors of $0.3 \%$ over $4000 \mathrm{~K}$.

\section{Observational inputs for the IRFM}

In the following paragraphs the different observational inputs which enter the application of the IRFM are commented on.

\subsection{IR monochromatic fluxes}

The determination of monochromatic fluxes at wavelengths of the IR continuum requires two observational inputs: Firstly, the measurement of IR photometry for the 
Table 1. Calibration of $q$ - and $R$-factors versus metallicity, $\log (g)$ and effective temperature for $\lambda_{\text {eff }}=1272.5 \mathrm{~nm}(J$ band $)$, computed using fluxes generated with Kurucz (1993) models

\begin{tabular}{|c|c|c|c|c|c|c|c|c|c|c|c|c|c|}
\hline & & $\mathrm{Fe} / \mathrm{H}$ & {$[=0.50$} & $\overline{\mathrm{Fe} / \mathrm{H}}$ & {[]$=0.00$} & $\mathrm{Fe} / \mathrm{H}$ & 00 & $\mathrm{Fe} / \mathrm{H}$ & 00 & $\mathrm{Fe} / \mathrm{H}$ & 00 & $\mathrm{Fe} / \mathrm{H}$ & \\
\hline $\bar{T} \overline{\text { eff }}$ & $\log (g)$ & $q_{J}$ & $\log \left(R_{J}\right)$ & $\overline{q_{J}}$ & $\log \left(R_{J}\right)$ & $\overline{q_{J}}$ & $\log \left(R_{J}\right)$ & $\overline{q J}$ & $\log \left(R_{J}\right)$ & $\overline{q J}$ & $\log \left(R_{J}\right)$ & $\overline{q J}$ & $\log \left(R_{J}\right)$ \\
\hline 3500 & 0.0 & 129 & & 1.0093 & & 050 & & 361 & & & & & 17282 \\
\hline 50 & 0.0 & & 904 & 106 & 3.25793 & 085 & 27145 & 82 & 031 & 0085 & 90 & 88 & \\
\hline 4000 & 0.0 & 55 & 3.24771 & 130 & 3.26872 & 105 & 3.27989 & 085 & 6091 & 1.0080 & 3.24966 & 082 & \\
\hline 4250 & 0.0 & 165 & 3.26798 & 1.0150 & 3.28540 & 1.0114 & 781 & 0084 & 3.28732 & 1.0082 & 3382 & .0076 & 3.29357 \\
\hline 4500 & 0.0 & 173 & 3.29582 & 1.0159 & 3.31095 & 0113 & 3.32382 & 084 & 3.32541 & 1.0078 & 664 & 0077 & .32698 \\
\hline 4750 & 0.0 & 181 & 3.32971 & 1.0166 & 3.34234 & 1.0111 & 3.35573 & 093 & 046 & 0083 & & 080 & $I$ \\
\hline 000 & 0.0 & & 3.36656 & 1.0170 & 3.37803 & 1.0108 & & 1.0091 & 3.39825 & 1.0083 & & 0081 & .40179 \\
\hline 5250 & 0.0 & & 40483 & 166 & 3.41549 & & 963 & 0089 & 3.43728 & 0083 & 3.44029 & 0084 & .44084 \\
\hline 5500 & 0.0 & & 63 & 1.01 & 5 & & 33 & 0 & 3 & 0085 & 3.47976 & 86 & 024 \\
\hline 0 & 0 & & 27 & 62 & 71 & & 72 & & 24 & 89 & 3.52226 & 0080 & 29 \\
\hline 00 & .0 & & 15 & 51 & 73 & & & 90 & 14 & 086 & & & \\
\hline 00 & 0 & & & & & & & & & & & & \\
\hline 50 & 1.0 & 26 & 23549 & 093 & 427 & 074 & & 070 & & 0076 & & 086 & 48 \\
\hline 4000 & 1.0 & 141 & 3.25985 & 1.0113 & 3.28051 & 1.0098 & 01 & 080 & 221 & 0076 & 231 & 0083 & 835 \\
\hline 4250 & 1.0 & 151 & 3.28015 & 132 & 3.29730 & 106 & & 079 & 45 & 082 & 9971 & 080 & 6 \\
\hline 00 & 1.0 & & .30441 & 0145 & 3.32039 & & & 0081 & 3.34103 & 0076 & 3.34228 & 075 & 34290 \\
\hline 50 & 1.0 & & 3 & & 6 & & & & & & 14 & & 783 \\
\hline 00 & 1 & & 3 & & 12 & & & & 19 & & 75 & 78 & 52 \\
\hline 50 & 1 & 77 & 5 & 55 & 34 & 5 & & 8 & & 4 & 53 & 32 & 59 \\
\hline 0 & 1.0 & 31 & 17 & 60 & 41 & 02 & & 6 & 58 & 35 & 0 & .0086 & 72 \\
\hline 0 & 1.0 & & 09 & 1.0158 & 296 & 03 & & 0088 & 10 & 33 & 40 & .0084 & 02 \\
\hline 00 & 1.0 & & 033 & 154 & 278 & 107 & & & 37 & 86 & & 080 & 862 \\
\hline 6250 & 1.0 & & 39 & 48 & 272 & & & & & & & & \\
\hline 00 & 1.0 & & & & & & & & & & & & \\
\hline & 1.0 & & & & & & & & & & & & 40 \\
\hline 0 & 1.0 & & & & 63 & & & & 44 & & & & 12 \\
\hline 00 & 2.0 & & & & 36 & & & & & 5 & & 4 & 58 \\
\hline 50 & 2.0 & & 7 & 2 & 3.2 & & & 2 & 3.2 & 36 & & 66 & 82 \\
\hline 00 & 2.0 & 28 & 49 & 03 & 3.2 & & & 0 & 22 & 32 & 8 & 36 & 48 \\
\hline 50 & 2.0 & & 45 & 23 & 6 & 8 & & 3 & 0 & 81 & 27 & 82 & 170 \\
\hline 4500 & 2.0 & & 3.31 & 36 & 3.3 & 1 & & & & 32 & 33 & 77 & 78 \\
\hline 50 & 2.0 & & & & & & & & & & & & \\
\hline & & & & & & & & & & & & & \\
\hline & 2 & & & & & & & & & & & & \\
\hline & 2 & & & & & & & & & & & & 64 \\
\hline & 2 & & & & & & & & & & & & 11 \\
\hline 00 & 2.0 & & & & & & & & 13 & 87 & & 88 & 23 \\
\hline 50 & 2.0 & 71 & 06 & 45 & 26 & 03 & 3.5 & 39 & 74 & 1.0 & 75 & 1.0088 & 47 \\
\hline 00 & 2.0 & 71 & 00 & 39 & 28 & 6 & 3 & 0094 & 61 & 91 & 64 & 93 & 89 \\
\hline 50 & 2.0 & 62 & 15 & 1 & 442 & 1. & 3 & 088 & 30 & 33 & & 77 & 43 \\
\hline 00 & 2.0 & & & & & & & & & & & & \\
\hline & & & & & & & & & & & & 19 & 63 \\
\hline & & & & & & & & & & & & & \\
\hline & & & & & & & & & & & & & \\
\hline & & & & & & & & & & & & 88 & 56 \\
\hline & 3.0 & & 62 & & 35 & & 3.3 & & 42 & 83 & 3.3 & 1.0083 & 3.36002 \\
\hline & 3 & & 94 & & 6 & & 3 & 2 & 83 & 79 & 3.3 & 1.0079 & 364 \\
\hline 5 & 3.0 & & 59 & 140 & 0 & 100 & 3.4 & - & 55 & 4 & 3. & .0077 & 3.4 \\
\hline 5 & 3 & & & & & & & & & 77 & & 77 & 354 \\
\hline 5500 & 3.0 & & 16 & & 3.4 & & & & 3. & 79 & & 78 & 349 \\
\hline & 3.0 & & & & & & & & & & & & 53 \\
\hline & 3.0 & & & & & & & & & & & & 25 \\
\hline & 3.0 & & & & & & & & & & & & 42 \\
\hline & 3.0 & & & & & & & & & & & & 241 \\
\hline & & & & & & & & & & & & 89 & 3.66511 \\
\hline 7000 & 3.0 & & & & & & & & & 1.0 & 3.69821 & 1.0079 & \\
\hline
\end{tabular}

problem stars with respect to a standard, and secondly the absolute flux calibration of the standard star. Then, entering the IR magnitudes measured in Eq. (2), we obtain monochromatic fluxes at $\lambda_{J}, \lambda_{H}, \lambda_{K}$ and $\lambda_{L^{\prime}}$ for each star in the sample.

In Paper II, a semi-empirical method was devised to determine the absolute calibration of the flux of Vega in the near-IR (from $J$ to $L^{\prime}$ ). This absolute calibration places temperatures derived applying the IRFM with Kurucz's models on the same scale as mean direct temperatures derived from angular diameter measurements.
It is worth noting the good agreement (within 1\%) with the semi-empirical calibration for Vega provided by Walker \& Cohen (1992), the theoretical one by Dreiling \& Bell (1980) and the "self-consistent" calibration by Blackwell et al. (1990).

The errors in the absolute IR flux calibration have different effects on the temperatures derived by mean of the IRFM, depending on the photometric band. The errors in the absolute IR flux calibration were estimated at $3 \%$ in the $J$ band, $4 \%$ in $H$ and $K$ bands and $5 \%$ in $L^{\prime}$ band (Paper II). Over $4000 \mathrm{~K}$, the net effect of 
Table 2. The same as in Table 1 for $\lambda_{\text {eff }}=1635.0 \mathrm{~nm}$ ( $H$ band $)$

\begin{tabular}{|c|c|c|c|c|c|c|c|c|c|c|c|c|c|}
\hline & & $\mathrm{Fe} / \mathrm{H}$ & $=0.50$ & $\mathrm{Fe} / \mathrm{H}$ & $\mathrm{I}]=0.00$ & $\overline{\mathrm{Fe} / \mathrm{H}}$ & $=-1.00$ & $\overline{\mathrm{Fe}} / \mathrm{H}$ & $=-2.00$ & $\mathrm{Fe} / \mathrm{H}$ & ]$=-3.00$ & {$[\mathrm{Fe} / \mathrm{H}]$} & $=-3.50$ \\
\hline$\overline{T_{\text {eff }}}$ & $\log (g)$ & $\frac{1}{q_{H}}$ & $\log \left(R_{H}\right)$ & $\frac{1}{q_{H}}$ & $\log \left(R_{H}\right)$ & $q_{H}$ & $\log \left(R_{H}\right)$ & $q_{H}$ & $\log \left(R_{H}\right)$ & $q_{H}$ & $\log \left(R_{H}\right)$ & $q_{H}$ & $\log \left(R_{H}\right)$ \\
\hline 3500 & 0.0 & 0809 & 3.24181 & 1014 & 3.19886 & 1090 & 3.21063 & 1.0985 & 3.20598 & 0831 & 3.21060 & 1.0752 & 3.19415 \\
\hline 3750 & 0.0 & 993 & 3.31560 & 0831 & 3.27487 & 0880 & 3.28193 & 1.0790 & 3.27814 & 0682 & 3.28650 & 1.0642 & 29334 \\
\hline 4000 & 0.0 & 587 & 3.38351 & 0669 & 3.34713 & 0709 & 3.35102 & 1.0646 & 3.34561 & 1.0587 & 35524 & .0575 & 35641 \\
\hline 4250 & 0.0 & 499 & 3.44632 & 1.0538 & 3.41665 & 0579 & 3.41760 & 1.0539 & 3.41784 & 1.0521 & 3.42171 & .0502 & 13181 \\
\hline 4500 & 0.0 & & 29 & 142 & 107 & 169 & 53 & & 68 & & 191 & 22 & 28 \\
\hline & 0.0 & & & & & & & & & & & & \\
\hline 00 & 0.0 & & & & & & & & & & & & \\
\hline 5250 & 0.0 & & & & & & & & & & & & \\
\hline 5500 & 0.0 & 75 & 3.73124 & 285 & 47 & 318 & 755 & 287 & 48 & 285 & 26 & 285 & 61 \\
\hline 5750 & 0.0 & 257 & 3.78833 & 1.0269 & 3.78763 & 1.0300 & 3.78920 & 305 & 65 & 303 & 365 & 303 & 06 \\
\hline 6000 & 0.0 & 1.0246 & 3.84500 & 0255 & 3.84644 & 0278 & 3.84964 & 1.0280 & 3.85264 & 1.0279 & 5424 & .0279 & 457 \\
\hline 3500 & 1.0 & 923 & 3.21626 & 1051 & 3.19139 & 1109 & 3.20771 & 1.1006 & 3.22724 & 1.0799 & 3.21766 & 1.0665 & 3.24775 \\
\hline 3750 & 1.0 & & 3.29 & 34 & 7 & 12 & 3.27986 & 17 & 3.29795 & 82 & 984 & $\$ 14$ & 3.29757 \\
\hline 4000 & 1.0 & & 6 & 26 & 3 & 27 & 55 & 69 & 33 & 81 & 83 & 53 & 367 \\
\hline 50 & 1.0 & & 22 & & & & & & 32 & & 26 & & 97 \\
\hline 0 & 1.0 & & 70 & 37 & 715 & & & & & & & & 05 \\
\hline 4750 & 1.0 & & 16 & & & & & & & & & 27 & 173 \\
\hline 5000 & 1.0 & & & & & & & & & & & & \\
\hline 5250 & 1.0 & & 87 & & & & & & & & & & \\
\hline 5500 & 1.0 & & 3.72321 & & & & & & & & & & \\
\hline 5750 & 1.0 & & 3.77210 & & 66 & & & & & & & & 900 \\
\hline 00 & 1.0 & & 0 & 7 & & 5 & 29 & & & & & 67 & 235 \\
\hline 50 & .0 & & & 7 & & & & & & & & 90 & 51 \\
\hline 00 & 1.0 & 9 & 94 & 262 & 306 & 77 & & 77 & & 77 & 27 & 77 & 66 \\
\hline 50 & .0 & 7 & 07 & 58 & 6 & 98 & 3. & 66 & 8 & 56 & 32 & 66 & 66 \\
\hline 7000 & .0 & 56 & 4.02783 & 0254 & 4.0 & 50 & 3 & 57 & 94 & 57 & 68 & 57 & 505 \\
\hline 3500 & .0 & & 60 & & & & & & & & & & 51 \\
\hline 3750 & 0 & & 27910 & 0878 & 91 & 90 & & 1.0795 & & & & 1.0486 & 027 \\
\hline 4000 & 0 & & & & & & & & & & & & \\
\hline & 0 & & & & & & & & & & & & \\
\hline & 0 & & & & & & & & & & & & 73 \\
\hline & .0 & & & & & & & & & & & & 75 \\
\hline 00 & 0 & & 5 & 6 & & 0 & & 4 & 38 & 5 & 66 & 71 & 369 \\
\hline 5250 & .0 & 2 & 16 & 97 & 3. & 30 & 8 & 9 & 01 & 36 & 76 & 36 & 38 \\
\hline 5500 & 0 & & & 72 & & 7 & & & & & & & 33 \\
\hline 5750 & 2.0 & 233 & 3.77057 & 0246 & 3.77239 & 1.0273 & 3.77954 & 1.0278 & 185 & 1.0 & 91 & 1.0280 & 3.78737 \\
\hline 6000 & .0 & & & & & & & & & & & 60 & 836 \\
\hline 0 & .0 & & & & & & & & & & & 37 & 23 \\
\hline & 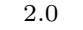 & & & & & & & & & & & 32 & 93 \\
\hline & 0 & & & & & & & & & & & & \\
\hline & 0 & & & & & & & & & & & 30 & 40 \\
\hline & .0 & & & & & & & & & & & 33 & 12 \\
\hline 3 & .0 & & 3 & 880 & & 9 & & 6 & & 4 & & 07 & 47 \\
\hline 4( & 0 & 4 & 14 & 44 & 3.3 & 26 & 6 & 4 & 8 & & & 72 & 09 \\
\hline 4250 & 0 & & 99 & 0618 & & 7 & & & & 25 & 20 & 395 & 9944 \\
\hline 4500 & 3.0 & & 07 & & & 96 & & & & 96 & & 387 & 3.53958 \\
\hline & .0 & & & & & & & & & & & 69 & 86 \\
\hline & .0 & & & & & & & & & & & & \\
\hline & .0 & & & & & & & & & & & & \\
\hline & 0 & & & & & & & & & & & & 990 \\
\hline & 3 & & & & & & & & & & & & \\
\hline & .0 & & & & & 7 & & & & & & 29 & \\
\hline & .0 & & & 17 & & 3 & & 9 & & 2 & & 02 & 723 \\
\hline 0 & 3.0 & 149 & 91474 & 1.0154 & 3.92003 & 1.0173 & 3.92756 & 1.0178 & 3.93182 & 1.0180 & 3371 & 1.0179 & 3.93428 \\
\hline 6750 & 3.0 & & 3.96113 & 1.0144 & 3.96615 & 1.0163 & 3.97360 & 1.0164 & 3.97767 & & & 1.0164 & 3.97989 \\
\hline 7000 & 3.0 & 1.0134 & 4.00836 & .0133 & 4.01298 & 1.0155 & 4.02025 & 1.0157 & 4.02417 & 1.0152 & 4.02442 & 1.0151 & 4.02561 \\
\hline
\end{tabular}

this systematic uncertainty is a drift of the temperature scale. If it happens that the above errors correlate in the three (four) bands, the maximum possible variation of the temperature scale would amounts to from $1.2 \%$ at $8000 \mathrm{~K}$ to $1.7 \%$ at $4000 \mathrm{~K}(1.3-1.7 \%)$ over $4000 \mathrm{~K}$. However, if as it is more likely, the errors in the three (four) bands are uncorrelated, the net effect of the uncertainty of the absolute calibration would be an approximately constant shift in the zero point over the whole temperature range amounting to at most $0.9 \%$. Although the indeterminacy of the zero point of the scale is common to all kind of methods used to derive effective temperatures, the method adopted in Paper II to fix the absolute cali- bration of the flux in the near IR was designed in order to minimising this error.

The programme of broad-band photometry in the near-IR is described in Paper IV. $J, H, K$ (and $L^{\prime}$ ) magnitudes were measured for $70 \%$ of the stars in the sample, with a mean accuracy of the order of $0.01-0.02$ mag for $J, H, K$ and $0.04 \mathrm{mag}$ for $L^{\prime}$. For the remainder of the stars, the photometry was obtained from the literature and transformed to the system of the TCS in order to compute near-IR monochromatic fluxes.

The isolated effect of the photometric errors on the $T_{\text {eff }}$ determination can be inferred from Tables 1-4, taking Eq. (3) into account. 
Table 3. The same as in Table 1 for $\lambda_{\text {eff }}=2175.0 \mathrm{~nm}$ ( $K$ band $)$

\begin{tabular}{|c|c|c|c|c|c|c|c|c|c|c|c|c|c|}
\hline & & {$[\mathrm{Fe} / \mathrm{H}$} & 50 & $\mathrm{Fe} / \mathrm{H}$ & $\mathrm{I}]=0.00$ & $\mathrm{Fe} / \mathrm{H}$ & $=-1.00$ & $\mathrm{Fe} / \mathrm{H}$ & $=-2.00$ & $\mathrm{Fe} / \mathrm{H}$ & $\overline{=}=-3.00$ & $\mathrm{Fe} / \mathrm{H}$ & $=$ \\
\hline$T_{\text {eff }}$ & $\log (g)$ & $\overline{q_{K}}$ & $\log \left(R_{K}\right)$ & $\overline{q_{K}}$ & $\log \left(R_{K}\right)$ & $\overline{q_{K}}$ & $\overline{\log \left(R_{K}\right)}$ & $\overline{q_{K}}$ & $\log \left(R_{K}\right)$ & $q_{K}$ & $\log \left(R_{K}\right)$ & $\overline{q_{K}}$ & $\log \left(R_{K}\right)$ \\
\hline 3500 & 0.0 & 0575 & 3.60479 & .0560 & 3.60904 & .0512 & 3.60753 & 0450 & 3.60304 & $\longdiv { 3 7 3 }$ & 3.59442 & 3041 & 4.57645 \\
\hline 37 & 0 & & 3. & & & & & & & & & & \\
\hline 10 & & & & & & & & & & & & & \\
\hline 50 & 0.0 & 12 & 744 & 181 & 191 & 408 & 356 & 314 & 439 & 1.0209 & 657 & 193 & 93 \\
\hline 4500 & 0.0 & 174 & 3.90676 & 439 & 3.90454 & 360 & 725 & 237 & 087 & 1.0193 & 177 & 192 & 202 \\
\hline 4750 & 0.0 & 428 & 3.97313 & 390 & 3.97042 & 1.0292 & 3.97460 & 1.0202 & 3.97656 & 1.0194 & 806 & 195 & 825 \\
\hline 5000 & 0.0 & 1.0376 & 4.03674 & 0333 & 4.03772 & 1.0220 & 4.03935 & 1.0194 & 1177 & 1.0194 & 1323 & 195 & 342 \\
\hline 5250 & .0 & 318 & 4.09924 & 0266 & 4.09965 & 1.0200 & 4.10222 & 1.0194 & 4.10575 & 1.0195 & 0716 & .0198 & 6625 \\
\hline 5500 & 0.0 & 54 & 927 & & 846 & 197 & 66 & 96 & 4.16788 & 1.0199 & 23 & 200 & 842 \\
\hline 5750 & 0.0 & 98 & 21790 & 2 & 4.21946 & 4 & 4.22438 & 2 & 4.22767 & 94 & 831 & 3 & 2872 \\
\hline 00 & .0 & & 30 & & 77 & & & & 316 & & 975 & & 016 \\
\hline 00 & .0 & & & & & & & & 70 & & 88 & & \\
\hline 3750 & 1.0 & & 55 & & & & & & 74 & & & & \\
\hline 4000 & 1.0 & & 14 & 70 & 35 & 07 & & & 845 & 60 & 22 & & 317 \\
\hline 4250 & 1.0 & & 196 & 437 & 202 & 369 & & & & 1.0215 & 874 & 200 & 423 \\
\hline 4500 & 1.0 & 132 & 080 & 402 & 116 & 334 & 077 & & 879 & 95 & 257 & 193 & \\
\hline 750 & .0 & & 660 & & 035 & & & & & & & & 3578 \\
\hline 00 & .0 & & & & & & & & & & & & 727 \\
\hline 50 & . 0 & & 71 & & 2 & & & & 65 & & & & 52 \\
\hline 00 & 0 & & & 4 & 3 & & & & 88 & & & & 378 \\
\hline 50 & .0 & 02 & 84 & 4 & 14 & 3 & 6 & & 5 & 4 & 99 & 92 & 157 \\
\hline 6000 & .0 & 78 & 27 & 3 & 4.2 & 3 & 4. & & 2 & 39 & 33 & 38 & 001 \\
\hline 6250 & 1.0 & 170 & 618 & 169 & 4.31996 & 180 & 53 & & 39 & 179 & 215 & 178 & 4.33256 \\
\hline 6500 & .0 & & & & & & & & & & & & 667 \\
\hline 50 & 0 & & & & & & & & & & & & \\
\hline & 0 & & & & & & & & & & & & \\
\hline & .0 & & & & & & & & & & & & 03 \\
\hline 50 & .0 & & & & & & & & & & & & 49 \\
\hline 00 & .0 & & & & & & & & 16 & 40 & & & 95 \\
\hline 50 & .0 & & 89 & & & & & & 84 & 20 & 90 & 06 & 68 \\
\hline 4500 & 2.0 & 992 & 65 & 365 & 55 & 308 & 276 & & 59 & 01 & 72 & 96 & 141 \\
\hline 4750 & .0 & 55 & 720 & 0329 & 92 & 0274 & & & & 91 & & 92 & 412 \\
\hline 5000 & .0 & 9 & & 5 & 4.0 & 30 & & & & & & & 16 \\
\hline 0 & .0 & & & & & & & & & & & & 38 \\
\hline & 0 & & & & & & & & & & & & \\
\hline & 0 & & & & & & & & & & & & 16 \\
\hline 0 & 0 & & & & & & & & & & 57 & & 13 \\
\hline 0 & 0 & & & & & & & & 42 & & 39 & & 381 \\
\hline 00 & .0 & & & & 57 & & 19 & & 41 & 72 & 75 & 69 & 7494 \\
\hline 50 & .0 & 35 & 55 & 4 & 4.4 & 0 & & & 4.2 & 1. & 88 & & 2824 \\
\hline 7000 & .0 & 32 & 99 & 130 & 4.4 & 47 & 4 & & 37 & 1. & 92 & 45 & 7727 \\
\hline 00 & .0 & 25 & 7 & & 69 & 7 & & & & & 51 & & 95 \\
\hline & 0 & & & & & & & & & & & & 391 \\
\hline & .0 & & & & & & & & & & & & 98 \\
\hline & .0 & & & & & & & & & & & & \\
\hline & 0 & & & & & & & & & & & & \\
\hline & 0 & & & & & & & & & & & & 03 \\
\hline & .0 & & & 71 & 4. & 26 & 31 & & 02 & 1. & 98 & & 781 \\
\hline 5 & .0 & 5 & 19 & 238 & 40 & 200 & 4 & & 61 & 1. & 75 & 95 & 338 \\
\hline 5500 & 0 & 18 & 5 & 0203 & 4.14699 & 1.0187 & 4.15799 & 9 & 4.16423 & 1.0191 & 3716 & 190 & 784 \\
\hline & .0 & & & & & 83 & & & 35 & & & & \\
\hline 6000 & 3.0 & & 084 & 0160 & 4.2 & 1.0176 & 332 & & 4.26896 & 1.0175 & 279 & 76 & 4.27334 \\
\hline 50 & 3.0 & & & 0 & 4.3 & 66 & 81 & & & & 230 & & 280 \\
\hline & 3.0 & & & & & & & & & & 56 & & 104 \\
\hline & 3.0 & & & & 4.4 & & & & & & & & 4.41777 \\
\hline 7000 & 3.0 & 04 & 45033 & 37 & 4.45569 & 1.0101 & 18 & & 4.46980 & 1.0134 & 396 & 1.0131 & 4.46566 \\
\hline
\end{tabular}

\subsection{Bolometric fluxes}

For the range of spectral types studied in the present work, nearly all the flux arriving at the earth atmosphere passes through the atmospheric windows. When comparing $F_{\text {Bol }}$ derived directly from calibrated spectra to $F_{\mathrm{Bol}}$ obtained by integrating $U B V R I$ photometry, Petford et al. (1988) report an accuracy of the order of $2 \%$. Therefore, the bolometric flux might be obtained for each star in the sample from broad-band photometry. However, photometric calibrations of the type provided by Blackwell \& Petford (1991) and Blackwell \& Lynas-Gray (1998) represent a more practical and accurate approach. We have obtained calibrations of this kind by fitting the bolometric fluxes of 184 stars of the sample, well distributed in metallicity, as a function of $K,(V-K)$ and $[\mathrm{Fe} / \mathrm{H}]$. Bolometric fluxes $\left(F_{\mathrm{Bol}}\right)$ have been obtained by integrating $U B V R I J H K$ photometry. The percentage of $F_{\text {Bol }}$ measured in $U-K$ bands ranges from $80 \%$ to $90 \%$ for the giant stars contained in our sample. The energy outside that wavelength range (i.e. UV and far IR flux) has been estimated with the help of models as described thoroughly in Paper III. The low dispersion of the fits grants the overall level of accuracy expected for the final temperatures derived in 

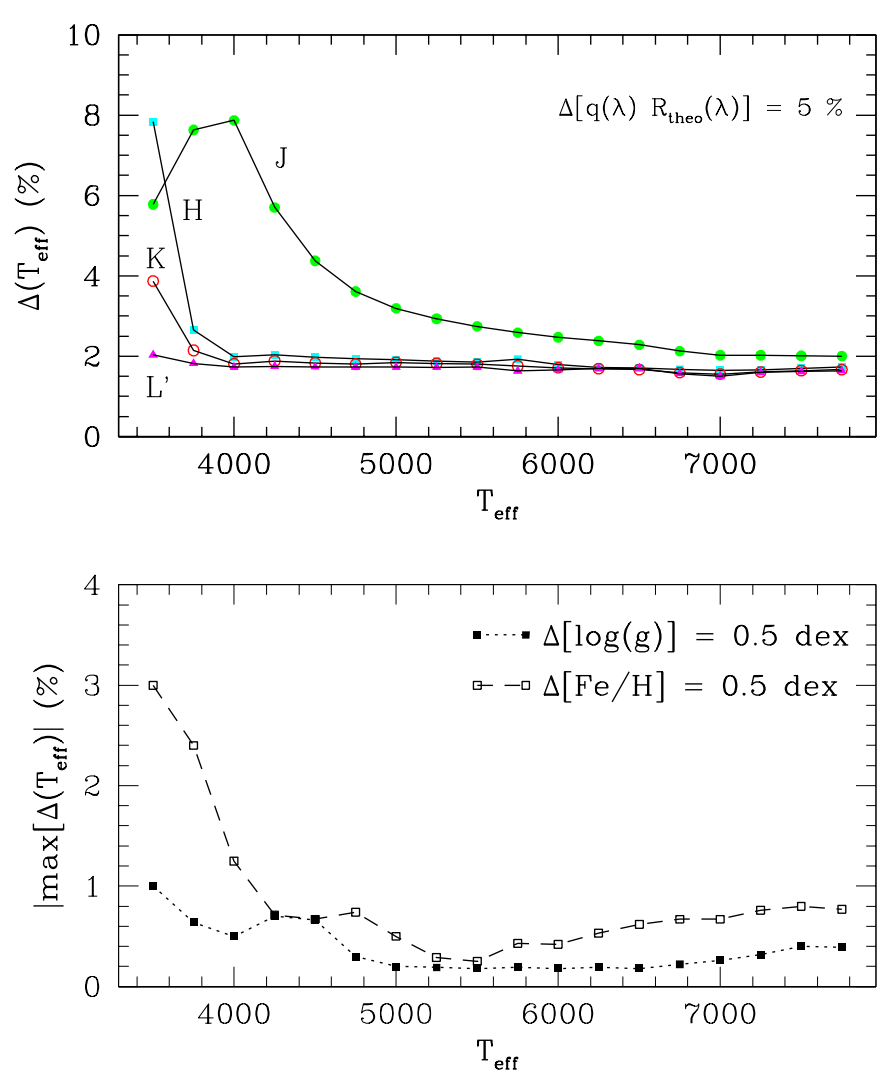

Fig. 2. Top: Mean uncertainties in the IRFM temperatures derived from $J, H, K$ and $L^{\prime}$ fluxes, induced by an error of $5 \%$ in the observational quotient $\frac{F_{\mathrm{Bol}}}{F_{\mathrm{cal}}\left(\lambda_{\mathrm{IR}}\right) 10^{-0.4\left(m-m_{\mathrm{cal}}\right)}}$. Bottom: Maximum mean uncertainties of the IRFM temperatures induced by an error of 0.5 dex in $[\mathrm{Fe} / \mathrm{H}]$ and $\log (g)$ respectively. Note the change in the scale of the ordinate axis with respect to the top figure

this work ${ }^{2}$. The result of the calibration for $(V-K) \leq 2.1$ is

$$
\begin{array}{r}
\log (\Phi(K))=-4.5939829-0.4560143(V-K) \\
-3.25133010^{-2}[\mathrm{Fe} / \mathrm{H}] \\
+1.116012110^{-2}[\mathrm{Fe} / \mathrm{H}](V-K) \\
+4.611436210^{-2}(V-K)^{2} \\
\sigma=0.006 \approx 1.5 \%, 50 \text { stars. }
\end{array}
$$

If we consider only stars with $(V-K) \geq 2.0$, then

$$
\begin{array}{r}
\log (\Phi(K))=-4.6538559-0.4152091(V-K) \\
-1.620859010^{-2}[\mathrm{Fe} / \mathrm{H}] \\
+4.113310110^{-3}[\mathrm{Fe} / \mathrm{H}](V-K)
\end{array}
$$

\footnotetext{
${ }^{2}$ Hereafter in this section, $K$ and $(V-K)$ are in the TCS system. Transformations from TCS to other systems are well described in Paper IV, in particular for the Johnson system: $K_{\mathrm{TCS}}=K_{J}-0.042+0.019(J-K)_{J}$, and $(V-K)_{\mathrm{TCS}}=0.050+0.993(V-K)_{J}$.
}
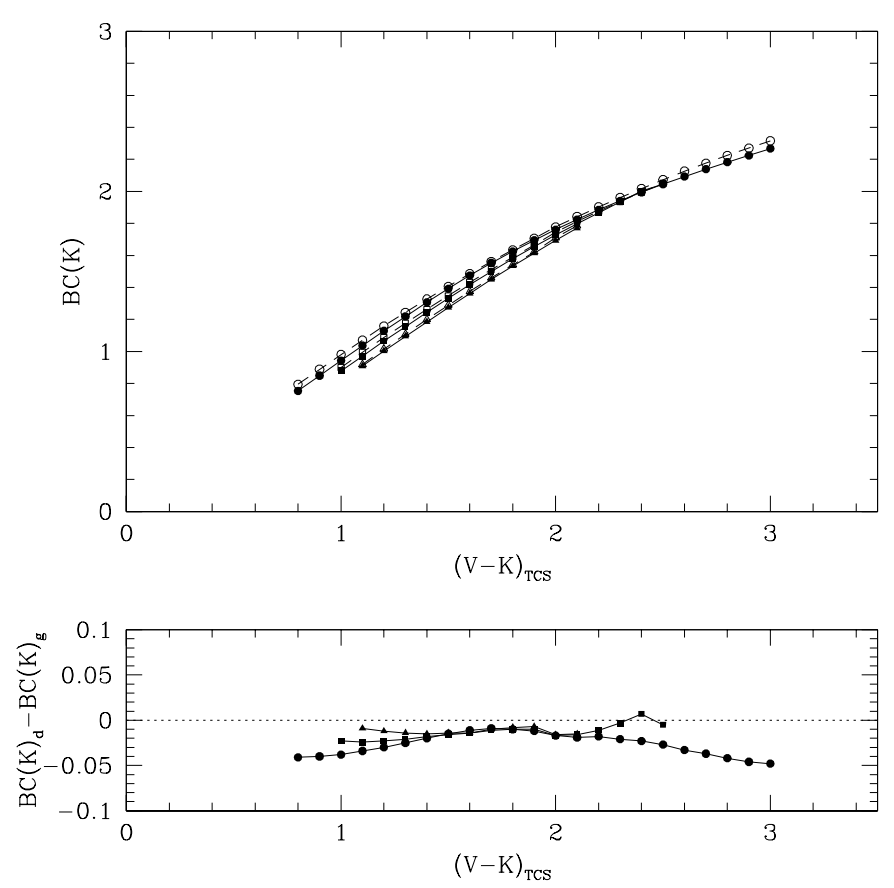

Fig. 3. Top: Bolometric correction to $K_{\mathrm{TCS}}$ versus $(V-K)_{\mathrm{TCS}}$. Solid circles correspond to dwarf stars and open circles to giant stars. Circles: $[\mathrm{Fe} / \mathrm{H}]=0.0$; squares: $[\mathrm{Fe} / \mathrm{H}]=-1.5$; triangles: $[\mathrm{Fe} / \mathrm{H}]=-3.0$. Bottom: Difference between the bolometric correction to $K_{\mathrm{TCS}}$ of dwarf and giant stars

$$
\begin{gathered}
+4.021384510^{-2}(V-K)^{2} \\
\sigma=0.006 \approx 1.5 \%, 141 \text { stars },
\end{gathered}
$$

where $\Phi(K)$ is the so-called reduced flux to magnitude $K$, defined through the equation $F_{\mathrm{Bol}}=10^{-0.4 K} \Phi(K)$. It should be noted that these calibrations are ultimately based on the optical absolute flux calibrations of Vega by Hayes \& Latham (1975) and Tüg et al. (1977), and the IR absolute flux measured in Paper II.

The corresponding bolometric correction for $K_{\mathrm{TCS}}$ magnitudes for giant stars obtained from Eqs. (4) and (5) is listed in Table 5, where the validity range of the calibration, in colour and metallicity, is shown. The differences from the bolometric correction obtained by applying the same method to main sequence stars are in the range $0.01-0.05$ mag (Fig. 3).

In Fig. 4 we show the difference between bolometric corrections or fluxes derived here, and those from the following authors:

\section{Bessell, Castelli and Plez (1998; BCP98)}

This work comprehensively provides bolometric corrections synthesized from different grids of stellar model fluxes of solar metallicity. The comparison of the $\mathrm{BC}(K)$ scales in Fig. 4a shows a consistent agreement in the range 
Table 4. The same as in Table 1 for $\lambda_{\text {eff }}=3690.0 \mathrm{~nm}\left(L^{\prime}\right.$ band $)$

\begin{tabular}{|c|c|c|c|c|c|c|c|c|c|c|c|c|c|}
\hline & & \multicolumn{2}{|c|}{$[\mathrm{Fe} / \mathrm{H}]=0.50$} & \multicolumn{2}{|c|}{$[\mathrm{Fe} / \mathrm{H}]=0.00$} & \multicolumn{2}{|c|}{$[\mathrm{Fe} / \mathrm{H}]=-1.00$} & \multicolumn{2}{|c|}{$[\mathrm{Fe} / \mathrm{H}]=-2.00$} & \multicolumn{2}{|c|}{$[\mathrm{Fe} / \mathrm{H}]=-3.00$} & \multicolumn{2}{|c|}{$[\mathrm{Fe} / \mathrm{H}]=-3.50$} \\
\hline$\overline{T_{\text {eff }}}$ & $\log (g)$ & $\overline{q_{L^{\prime}}}$ & $\log \left(R_{L^{\prime}}\right)$ & $q_{L^{\prime}}$ & $\log \left(R_{L^{\prime}}\right)$ & $q_{L^{\prime}}$ & $\log \left(R_{L^{\prime}}\right)$ & $\overline{q_{L^{\prime}}}$ & $\log \left(R_{L^{\prime}}\right)$ & $\overline{q_{L^{\prime}}}$ & $\log \left(R_{L^{\prime}}\right)$ & $q_{L^{\prime}}$ & $\log \left(R_{L^{\prime}}\right)$ \\
\hline 3500 & 0.0 & .9980 & 4.43201 & 1.0002 & 4.44019 & 1.0052 & 4.43689 & 0.9440 & 5.31112 & 1.0288 & 5.37874 & 1.0298 & 5.39615 \\
\hline 3750 & 0.0 & 964 & 4.52257 & 0.9991 & 4.52605 & 1.0050 & 4.51965 & 0.9489 & 5.36607 & 1.0253 & 5.32900 & 1.0253 & 31477 \\
\hline 4000 & 0.0 & 946 & 60545 & 978 & 4.60534 & 043 & 9802 & 0.9553 & 304 & 22 & 9304 & 0209 & 871 \\
\hline 4250 & 0.0 & 942 & .68165 & 977 & 4.67927 & 0041 & 4.67238 & 0.9634 & 5.40786 & 73 & 1546 & 84 & 022 \\
\hline 4500 & 0.0 & 941 & .75293 & 977 & 4.74959 & 1.0048 & 4.74428 & 1.0069 & 4.74289 & 1.0070 & 4.74369 & 1.0075 & 74357 \\
\hline 4750 & 0.0 & 0.9946 & 4.81979 & 0.9985 & 4.81548 & 1.0047 & 4.81257 & 1.0061 & 4.81335 & 1.0065 & 4.81357 & 1.0070 & 4.81353 \\
\hline 5000 & 0.0 & 0.9953 & 4.88300 & 0.9989 & 4.88035 & 1.0046 & 4.87812 & 1.0059 & 4.88024 & 1.0062 & 4.88086 & 1.0062 & 108 \\
\hline 5250 & 0.0 & 0.9951 & 4.94791 & 0.9992 & 4.94149 & 1.0041 & 4.94215 & 1.0053 & 4.94438 & 1.0056 & 4.94559 & 1.0052 & 598 \\
\hline 5500 & 0.0 & 0.9957 & 5.00739 & 0.9986 & 5.00660 & 1.0024 & 5.00829 & 1.0045 & 5.00590 & 1.0045 & 5.00727 & 1.0045 & 5.00739 \\
\hline 5750 & 0.0 & 961 & 5.06580 & 9988 & 5.06629 & 0022 & 5.06964 & 1.0033 & 5.07258 & 1.0035 & 5.07412 & .0033 & 07461 \\
\hline 6000 & 0.0 & 63 & .12282 & 984 & 5.12511 & 1.0016 & 5.12965 & 1.0025 & 5.13276 & 1.0027 & 5.13432 & 1.0025 & 5.13481 \\
\hline 3500 & 1.0 & & 42786 & & 3654 & 063 & 19 & 0.9940 & 4.54230 & 1.0268 & 5.24524 & 0265 & 5.22568 \\
\hline 3750 & 1.0 & & 901 & & 441 & 553 & 58 & 1.0078 & 4.52446 & 1.0234 & 5.20838 & .0220 & 13232 \\
\hline 4000 & 1.0 & 57 & 294 & & 4.60478 & 50 & 371 & 1.0074 & 4.60319 & 0193 & 5.15761 & 0190 & 9787 \\
\hline 4250 & 1.0 & 947 & 4.67993 & 980 & 4.67964 & 1.0046 & 4.67839 & 1.0074 & 7864 & 1.0067 & 4.68711 & .0153 & 989 \\
\hline 4500 & 1.0 & 0.9944 & 4.75176 & 0.9981 & 4.75033 & 1.0044 & 4.74973 & 1.0073 & 4.74970 & 1.0078 & 4.75097 & 1.0075 & 4.75112 \\
\hline 4750 & 1.0 & 941 & 4.81947 & 0.9985 & 4.81814 & 1.0046 & 4.81692 & 1.0069 & 4.81882 & 1.0070 & 4.81943 & .0071 & 4.81955 \\
\hline 5000 & 1.0 & 945 & 4.88441 & 85 & 4.88207 & .0049 & 201 & 1.0067 & 8369 & 1.0067 & 3503 & 1.0067 & 8525 \\
\hline 5250 & 1.0 & 52 & 164 & 86 & 270 & 42 & 24 & 1.0055 & 562 & 1.0057 & 798 & 060 & 819 \\
\hline 5500 & 1.0 & 50 & 216 & 38 & 36 & 36 & 62 & 49 & 50 & 1.0052 & 317 & 055 & 831 \\
\hline 5750 & 1.0 & 48 & 358 & 984 & 5.05770 & 032 & 5.06060 & 1.0040 & 5406 & 44 & 547 & .0044 & 5.06585 \\
\hline 6000 & 1.0 & 45 & 5.11890 & 973 & 5.12022 & 007 & 90 & 1.0023 & 814 & 1.0025 & 313 & 025 & 261 \\
\hline 6250 & 1.0 & 9939 & 5.17333 & 0.9961 & 5.17565 & 9994 & 5.18060 & 1.0008 & 5.18379 & 1.0008 & 5.18547 & 1.0011 & 5.18575 \\
\hline 6500 & 1.0 & & 5.22647 & 54 & 5.22945 & 986 & 5.23480 & 0.9993 & & 0.9997 & 5.23945 & 0.9995 & 5.23994 \\
\hline 6750 & 1.0 & & 5.2 & & 89 & & & & & & & & \\
\hline 7000 & .0 & & 4 & & & & & & & 75 & 85 & 76 & 11 \\
\hline 3500 & 2.0 & & 33 & & 20 & 66 & & 96 & 79 & 40 & 305 & 231 & 232 \\
\hline 750 & 2.0 & & 96 & 16 & 315 & 61 & 02 & 83 & 36 & 99 & 210 & 190 & 3957 \\
\hline 4000 & 2.0 & 71 & 8 & 01 & 21 & 56 & 55 & 79 & 28 & 86 & 20 & 91 & 300 \\
\hline 4250 & 2.0 & 56 & 997 & 993 & 087 & 50 & 84 & 1.0076 & 248 & 1.0086 & 497 & 882 & 3612 \\
\hline 4500 & 2.0 & 0.9952 & 4.75207 & 0.9988 & 4.75191 & 1.0044 & 4.75252 & 1.0078 & 5424 & 1.0079 & 586 & 082 & 4.75593 \\
\hline 4750 & 2.0 & 0.9948 & 4.82019 & 0.9986 & 4.81920 & 1.0047 & 935 & 1.0072 & 4.82218 & 1.0080 & 302 & 078 & 4.82340 \\
\hline 5000 & 2.0 & 950 & 4.88469 & & 313 & 51 & 98 & 1. & 32 & 74 & 83 & 71 & 56 \\
\hline 5250 & .0 & & & 5 & & 52 & & 1. & & 67 & 03 & 66 & 54 \\
\hline 0 & 2.0 & & & & & & & & & & 16 & 64 & 63 \\
\hline & 2.0 & & & & & & & & & & 17 & 50 & 660 \\
\hline 00 & 2.0 & & 22 & 37 & 12 & 32 & 21 & 40 & 96 & 46 & 50 & 945 & 096 \\
\hline 6250 & 2.0 & 45 & 999 & 80 & 360 & 18 & 37 & 1.0031 & 173 & 1.0033 & 350 & 1.0031 & 5.17391 \\
\hline 6500 & 2.0 & 941 & .22146 & 961 & 5.22437 & 998 & 5.23000 & 1.0007 & 5.23372 & 1.0011 & 5.22597 & 1.0011 & 5.22834 \\
\hline 6750 & 2.0 & 0.9933 & 5.27227 & 0.9950 & 5.27539 & 83 & 112 & 0.9 & 8438 & 0.9992 & 5.28613 & 995 & 8638 \\
\hline 7000 & 2.0 & 0.9923 & 5.32181 & 0.9941 & 5.32526 & .9967 & 5.33092 & 0.9973 & 5.33412 & 0.9976 & 45 & 0.9979 & 3568 \\
\hline 3500 & 3.0 & 21 & & 42 & 69 & 99 & 46 & 96 & 31 & 20 & 45 & 09 & 991 \\
\hline 3750 & 3.0 & 00 & 4.5 & 1.0034 & 4.5 & 1.0075 & 29 & $1 .($ & 4.5 & 1.0106 & 900 & 05 & 4.54992 \\
\hline 0 & 3.0 & & & & & & & $1 .($ & 4. & 94 & 13 & 03 & 211 \\
\hline & 3.0 & & & & & & & & & & & & 16 \\
\hline & 3.0 & & & & & 52 & 96 & 75 & 302 & 87 & 4.75800 & 1.0081 & 4.75900 \\
\hline 4750 & 3.0 & 3 & 993 & 93 & 89 & 51 & 03 & 976 & 4.82362 & 1.0082 & 4.82517 & 1.0079 & 4.82579 \\
\hline 5000 & 3.0 & 948 & 88470 & 995 & 385 & 1.0050 & 3527 & 1.0072 & 4.88775 & $1 .($ & 916 & 1.0078 & 4.88989 \\
\hline 5250 & 3.0 & 0.9953 & 4.94590 & 0.9994 & 4.94559 & 1.0053 & 4.94604 & 1.0073 & 4.94865 & 1.0074 & 4.95109 & 1.0078 & 4.95150 \\
\hline 5500 & 3.0 & 957 & .00461 & 0.9996 & 5.00336 & 1.0055 & 5.00450 & 1.0069 & 5.00809 & 1.0072 & 5.01022 & 1.0073 & 5.01069 \\
\hline 50 & 3.0 & 0.9956 & 5.05940 & 0.9996 & 5.05849 & 1.0051 & 5.06092 & 1.0062 & 5.06496 & 1.0065 & 5.06700 & 1.0066 & 5.06745 \\
\hline 6000 & 3.0 & 960 & 5.11158 & 0.9998 & 5.11139 & 1.0046 & 5.11537 & 1.0058 & 5.11931 & 1.0059 & 5.12131 & 1.0060 & 5.12176 \\
\hline 6250 & 3.0 & 959 & .16213 & 0.9999 & 5.16263 & 1.0037 & 5.16789 & 1.0049 & 5.17170 & 1.0051 & 5.17362 & 1.0050 & 5.17404 \\
\hline 00 & 3.0 & 1 & .21122 & 992 & 5.21296 & 026 & 360 & 1.0 & 2229 & 1.0040 & 5.22396 & 1.0041 & 5.22436 \\
\hline & 3.0 & & 5.26760 & 83 & 5.26248 & 1.0016 & 5.26782 & 1.0026 & 5.27123 & 1.0026 & 5.27283 & 1.0026 & 5.27324 \\
\hline 7000 & 3.0 & 0.9957 & 5.31547 & 0.9978 & 5.31901 & 1.0005 & 5.32567 & 1.0014 & 5.32951 & 1.0010 & 5.32118 & 1.0011 & 5.32229 \\
\hline
\end{tabular}

$(V-K)=1.4-3.6$. However, in the borders of the colour range of our calibration differences increase. The discrepancies at the cooler edge of the temperature scale point are probably connected with the limitations of Kurucz's (1993) models under $4500-4000 \mathrm{~K}$, however the reason for the discrepancy at the hotter edge is unclear.

\section{Blackwell and Lynas-Gray 1998 (BL98)}

This work presents a re-analysis of the results of Blackwell \& Petford (1991) by using the same techniques. In Fig. 3c, we show the ratio of our bolometric flux to that of BL98 versus temperature for 50 giants common to both works. The mean difference is $1.4 \%$ (BL98 fluxes greater) with a dispersion of $1.7 \%$. No appreciable trend with temperature is observed in the range $4000-8000 \mathrm{~K}$. The offset is probably related to the different absolute flux calibrations adopted.

\section{Flower (1996; F96)}

This study provides a thorough analysis of the bolometric correction based on measurements of 335 stars compiled from the literature. For 37 giants common with our sample (mainly Population I stars), we have transformed the $\mathrm{BC}(V)$ provided in Table 3 of $\mathrm{F} 96$ to $\mathrm{BC}(K)$ adopting the following parameters for the Sun: $\mathrm{BC}_{\odot}(V)=-0.12$ and $(V-K)_{\odot}=1.524$. From the comparison, two main 
Table 5. Bolometric correction to $K_{\mathrm{TCS}}$ magnitude versus $(V-K)_{\mathrm{TCS}}$

\begin{tabular}{c|cc|cc|cc|ccc}
\hline$(V-K)$ & \multicolumn{7}{|c}{$\mathrm{BC}(\mathrm{K})$} \\
\hline & {$[\mathrm{Fe} / \mathrm{H}]$} & $=0.00$ & {$[\mathrm{Fe} / \mathrm{H}]$} & $=-1.0$ & {$[\mathrm{Fe} / \mathrm{H}]$} & $=-2.0$ & {$[\mathrm{Fe} / \mathrm{H}]$} & $=-3.0$ \\
& dwarfs & giants & dwarfs & giants & dwarfs & giants & dwarfs & giants \\
\hline 0.800 & 0.754 & 0.795 & $(0.710)$ & $(0.736)$ & - & - & - & - \\
0.900 & 0.849 & 0.889 & 0.805 & 0.833 & - & - & - & - \\
1.000 & 0.943 & 0.981 & 0.899 & 0.928 & - & - & - & - \\
1.100 & 1.037 & 1.071 & 0.993 & 1.021 & $(0.950)$ & $(0.970)$ & $(0.910)$ & $(0.919)$ \\
1.200 & 1.129 & 1.159 & 1.085 & 1.111 & 1.043 & 1.063 & 1.003 & 1.015 \\
1.300 & 1.219 & 1.244 & 1.176 & 1.199 & 1.135 & 1.154 & 1.095 & 1.109 \\
1.400 & 1.307 & 1.327 & 1.265 & 1.284 & 1.224 & 1.242 & 1.185 & 1.200 \\
1.500 & 1.392 & 1.407 & 1.352 & 1.368 & 1.312 & 1.328 & 1.275 & 1.289 \\
1.600 & 1.475 & 1.486 & 1.436 & 1.449 & 1.398 & 1.412 & 1.362 & 1.376 \\
1.700 & 1.553 & 1.562 & 1.517 & 1.528 & 1.483 & 1.494 & 1.450 & 1.460 \\
1.800 & 1.625 & 1.635 & 1.594 & 1.604 & 1.563 & 1.573 & 1.534 & 1.542 \\
1.900 & 1.695 & 1.707 & 1.667 & 1.678 & 1.641 & 1.650 & 1.615 & 1.622 \\
2.000 & 1.761 & 1.778 & 1.738 & 1.755 & 1.716 & 1.732 & 1.694 & 1.710 \\
2.100 & 1.824 & 1.843 & 1.806 & 1.824 & 1.789 & 1.805 & 1.771 & 1.786 \\
2.200 & 1.885 & 1.903 & 1.872 & 1.885 & 1.860 & 1.868 & - & 1.850 \\
2.300 & 1.941 & 1.962 & 1.937 & 1.945 & 1.932 & 1.928 & - & 1.911 \\
2.400 & 1.995 & 2.018 & 2.000 & 2.003 & 1.995 & 1.987 & - & 1.971 \\
2.500 & 2.046 & 2.073 & $(2.046)$ & 2.058 & $(2.046)$ & 2.043 & - & 2.029 \\
2.600 & 2.093 & 2.126 & - & 2.112 & - & 2.098 & - & 2.084 \\
2.700 & 2.139 & 2.176 & - & 2.163 & - & 2.151 & - & 2.138 \\
2.800 & 2.183 & 2.225 & - & 2.213 & - & 2.201 & - & 2.189 \\
2.900 & 2.225 & 2.271 & - & 2.260 & - & 2.250 & - & 2.239 \\
3.000 & $(2.268)$ & 2.316 & - & 2.306 & - & 2.296 & - & $(2.287)$ \\
3.100 & - & 2.358 & - & 2.349 & - & $(2.341)$ & - & - \\
3.200 & - & 2.399 & - & 2.391 & - & - & - & - \\
3.300 & - & 2.437 & - & $(2.430)$ & - & - & - & - \\
3.400 & - & 2.473 & - & - & - & - & - & - \\
3.500 & - & 2.508 & - & - & - & - & - & - \\
3.600 & - & 2.540 & - & - & - & - & - & - \\
3.700 & - & 2.571 & - & - & - & - & - & - \\
3.800 & - & $(2.599)$ & - & - & - & - & - & - \\
\hline & & & & & & & & - \\
\hline
\end{tabular}

features can be observed in Fig. 4b. The differences show a conspicuous slope with colour. The probable reason for this trend is coupled with the different temperature scales adopted in both studies. Furthermore the scatter about the mean line of differences is slightly larger $(\approx 3 \%)$ than expected if one sums in quadrature the internal errors of both works. The explanation of this point, apart from the different temperature scales used implicity in the calculation, may be partly related to the inhomogeneity of the F96 sample and partly to the uncertainties introduced by the transformation from $\mathrm{BC}(V)$ to $\mathrm{BC}(K)$.

\section{Bell \& Gustaffson 1989 (BG89)}

This study is based on a method similar to that applied here using instead 13-colour, UV and broad-band near-IR photometry. There are 21 common stars whose fluxes appear to be shifted $6 \%$ with a dispersion of $1.7 \%$ (Fig. 4c) from ours. This difference is probably connected with the absolute flux calibration and the photometric calibration of the 13-colour system. Notice that BG89 fluxes are also shifted $4-5 \%$ with respect to those of BL98, so that the relative differences with our work are consistent.

In summary, the agreement of the bolometric fluxes derived here for solar metallicity stars is within the errorbars expected from the sources of uncertainty affecting the various methods: errors in the absolute calibrations adopted in the optical and IR ranges, differences in the atmosphere models grids. It is worth noting at this point that a $3 \%$ error on the bolometric flux implies a $1 \%$ error in the temperature derived by mean of the IRFM.

\subsection{The reddening correction}

A considerable number of the stars in the sample are distant from the solar neighbourhood $(D>300 \mathrm{pc})$, 
Table 6. Temperatures derived for the field stars of the sample. Column 1: Identification. The stars are ordered in right ascension. Column 2: Metallicity. Column 3: Surface gravity. Column 4: Bolometric flux in $10^{-2} \mathrm{erg} \mathrm{cm}^{-2} \mathrm{~s}^{-1}$. Column 5: Interstellar reddening. Column 6: Temperature derived in band $J$ (units are K). Column 7: Error in $T_{J}$ computed considering errors in $F_{\mathrm{Bol}}$, monochromatic fluxes, $\log (g)$ and $[\mathrm{Fe} / \mathrm{H}]$. Columns 8-9: The same as in Cols. 6-7 for temperature derived in band $H$. Columns 10-11: The same as in Cols. 6-7 for temperature derived in band $K$. Columns $12-13$ : The same as in Cols. 6-7 for temperature derived in band $L^{\prime}$. Column 14: The weighted mean temperature derived from $T_{J}, T_{H}, T_{K}$ and $T_{L^{\prime}}$. Column 15: Mean error computed by considering linear transmission of errors from Cols. 7, 9, 11 and 13. Column 16. Number of temperatures considered in the average of Col. 14

\begin{tabular}{|c|c|c|c|c|c|c|c|c|c|c|c|c|}
\hline ID & {$[\mathrm{Fe} / \mathrm{H}]$} & $\log (g)$ & $F_{\mathrm{Bol}}$ & $E(B-V)$ & $T_{J}$ & $\Delta T_{J} \quad T_{H}$ & $\Delta T_{H} \quad T_{K}$ & $\Delta T_{K}$ & $T_{L^{\prime}}$ & $\Delta T_{L^{\prime}}$ & $T_{\text {mean }}$ & $\frac{\Delta T_{\text {mean }} n}{843}$ \\
\hline SAO166037 & -1.66 & 2.00 & $7.286 \mathrm{E}-11$ & 0.008 & 5387 & 1135353 & 775327 & 71 & & 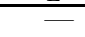 & 5351 & 843 \\
\hline SAO147079 & -1.38 & 0.00 & $4.436 \mathrm{E}-11$ & 0.000 & 5054 & 824945 & 574933 & 56 & - & - & 4968 & 63 \\
\hline SAO166160 & -1.12 & 2.00 & $4.592 \mathrm{E}-11$ & 0.015 & 5273 & 1175284 & 795244 & 66 & - & - & 5265 & 83 \\
\hline BS0080 & -0.16 & 2.10 & 3.450E-09 & 0.000 & 4276 & 1424182 & 464186 & 34 & - & - & 4184 & 39 \\
\hline BS0088 & 0.14 & 4.50 & $7.418 \mathrm{E}-10$ & 0.000 & 5741 & 1185765 & 985700 & 97 & - & - & 5735 & 103 \\
\hline SAO073973 & -0.11 & 2.50 & $1.807 \mathrm{E}-10$ & .020 & 7507 & 1847484 & 1527484 & 144 & - & - & 7491 & 158 \\
\hline SAO021465 & -1.92 & 2.40 & $3.149 \mathrm{E}-10$ & 065 & 4986 & 1074978 & 725000 & 61 & $\ldots$ & - & 4990 & 66 \\
\hline BS0114 & 0.00 & 3.00 & $2.006 \mathrm{E}-09$ & 0.000 & 6990 & 148 & -7006 & 113 & 6907 & 232 & 6979 & 200 \\
\hline SAO147321 & -2.35 & 1.41 & $1.353 \mathrm{E}-10$ & 0.000 & 4848 & 1054869 & 634866 & 53 & - & - & 4867 & 58 \\
\hline SAO074086 & -0.50 & 2.50 & $6.151 \mathrm{E}-10$ & 0.000 & 4717 & 1724797 & 704789 & 52 & - & - & 4792 & 60 \\
\hline D3008 & 1.85 & 1.50 & E-11 & 15 & $<3500$ & 3923 & 704047 & 60 & - & - & 4047 & 60 \\
\hline SAO166375 & 0.92 & 2.00 & $3.793 \mathrm{E}-11$ & 0.010 & 5244 & 1165239 & 755222 & 68 & - & - & 5233 & 82 \\
\hline BS0163 & -0.70 & 2.40 & $5.772 \mathrm{E}-09$ & 0.000 & 4837 & 1044890 & 674889 & 54 & 4962 & 58 & 4914 & 59 \\
\hline HR0165 & 0.02 & 1.95 & $2.067 \mathrm{E}-08$ & 000 & 4209 & 2424333 & 684326 & 38 & 4332 & 65 & 4329 & 53 \\
\hline HR0168 & -0.10 & 2.10 & $4.697 \mathrm{E}-08$ & 000 & 4557 & 1874591 & 704585 & 47 & 4568 & 70 & 4582 & 60 \\
\hline BS0219 & -0.22 & 4.40 & $1.122 \mathrm{E}-08$ & 000 & 5648 & 1645842 & 945818 & 89 & - & - & 5790 & 1073 \\
\hline SAO054175 & -1.28 & 2.00 & $3.835 \mathrm{E}-10$ & 045 & 4542 & 1014535 & 494568 & 41 & - & - & 4553 & 45 \\
\hline BS0253 & -0.25 & 1.75 & $5.055 \mathrm{E}-09$ & 30 & 4602 & 1304460 & 504447 & 40 & - & - & 4453 & 44 \\
\hline 170 & -1.90 & 2.00 & $469 \mathrm{E}-11$ & 19 & 5035 & 1185092 & 765119 & 59 & - & - & 5091 & 78 \\
\hline SAO036851 & -0.80 & 2.00 & $6.291 \mathrm{E}-10$ & 0.030 & 4996 & 1464941 & 834946 & 61 & - & - & 4944 & 70 \\
\hline 0 & 0.93 & .30 & & & & 65 & 882 & 53 & - & - & 068 & \\
\hline BS0316 & -0.40 & 2.40 & $1.090 \mathrm{E}-09$ & 00 & 4323 & 2644436 & 864407 & 51 & 4408 & 21 & 4412 & 38 \\
\hline & -0.91 & 1.50 & 966 & 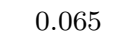 & 350 & 1174358 & 574395 & 38 & 4476 & 79 & 402 & 53 \\
\hline 0337 & 0.00 & 1.60 & 07 & 0 & & 2143767 & 3786 & 21 & 3775 & 49 & 3783 & 29 \\
\hline 43 & 0.00 & 2.00 & 4.56 & 00 & 7709 & 1737818 & 1547822 & 142 & - & - & 7787 & 155 \\
\hline 424 & -0.54 & 4.00 & $731 \mathrm{E}-11$ & 20 & & 1595635 & 945585 & - & - & 82 & 625 & 1033 \\
\hline & -0.15 & 2.80 & $280 \mathrm{E}-08$ & & 00 & 1374705 & 614668 & 46 & 4700 & 54 & 4689 & 533 \\
\hline 37 & 1.60 & 1.50 & 10 & & & 34527 & 4542 & 41 & - & 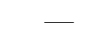 & 4535 & 452 \\
\hline SAO167076 & -1.50 & 2.00 & $157 \mathrm{E}-11$ & 0.010 & 4827 & 1104828 & 94842 & 54 & - & - & 1836 & 612 \\
\hline BS0434 & -0.39 & 1.90 & $6.326 \mathrm{E}-09$ & 0.000 & 4023 & 1424031 & 344054 & 26 & 4068 & 183 & 4046 & 413 \\
\hline & 99 & 34 & & & & 7 & & 88 & 58 & 18 & 783 & 112 \\
\hline 4 & -0.03 & 2.00 & 10 & & 4281 & 84386 & 4354 & 8 & 434 & 35 & 359 & 41 \\
\hline & 0.30 & 1.50 & 99 & & & 774104 & 18 & 30 & 4116 & 36 & 114 & 37 \\
\hline 437 & 1.60 & 1.50 & & & & 34527 & 42 & 41 & - & - & 335 & 45 \\
\hline 67076 & -1.50 & 2.00 & 11 & & 27 & 104828 & 94842 & 54 & — & - & 4836 & 61 \\
\hline 34 & -0.39 & 1.90 & & & & 24031 & 54 & 26 & 406 & 83 & 4046 & 41 \\
\hline & & 3.84 & & & & 95 & 49 & 8 & 58 & 218 & 83 & 1124 \\
\hline 64 & -0.03 & 2.00 & 88 & & & 1484386 & 4354 & 35 & 4348 & 35 & 4359 & $41:$ \\
\hline & -1.92 & 0.00 & & & & 984269 & 83 & 38 & - & - & 4277 & 452 \\
\hline BS0489 & -0.30 & 1.50 & $8.598 \mathrm{E}-09$ & 0.000 & 3976 & 1774104 & 14118 & 30 & 4116 & 36 & 4114 & 37 \\
\hline & .50 & & & & & 04 & & 49 & & 69 & & 60 \\
\hline & 0.25 & 1.05 & 08 & & & 5 & 77 & 34 & 42 & 63 & 4277 & 50 \\
\hline & 0.20 & 2.50 & & & & 44 & & 48 & 45 & 70 & 4490 & 61 \\
\hline & 01 & 4.0 & & & & 35 & 5 & 83 & 59 & 154 & 5906 & 111 \\
\hline 0395 & -2.00 & 1.7 & 11 & & & $4 \varepsilon$ & & 52 & $\ldots$ & - & 4815 & 58 \\
\hline & & 2. & & & & 2. & & 71 & - & - & & 67 \\
\hline & -0.43 & & & & & 24 & 87 & 45 & 4913 & 92 & 4815 & 61 \\
\hline B & -1.07 & 2.00 & & & & 14 & 00 & 54 & 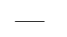 & - & 4902 & 59 \\
\hline & & 2.80 & & & & 44 & 03 & 43 & $45 \mathrm{~s}$ & 48 & 4608 & 48 \\
\hline BS0911 & .00 & 1.23 & $9.943 \mathrm{E}-08$ & 0.000 & 3547 & 1663628 & 423703 & 22 & 3709 & 185 & 3704 & 39 \\
\hline & & & & & & 548 & & 54 & & 78 & & 62 \\
\hline 889 & 0.00 & 2.0 & & & & 14543 & 444313 & 32 & - & - & 4315 & 37 \\
\hline & -1.70 & 2.00 & & & & 12248 & 04 & 53 & - & 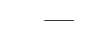 & & 60 \\
\hline & 11 & 2.0 & 5.48 & & & 9547 & 624 & 50 & - & - & 4781 & 55 \\
\hline BS1132 & -0.50 & 1.75 & $1.277 \mathrm{E}-09$ & 0.030 & 4761 & 1134812 & 724783 & 46 & 4814 & 113 & 4798 & 67 \\
\hline
\end{tabular}


Table 6. continued

\begin{tabular}{|c|c|c|c|c|c|c|c|c|c|c|c|c|c|}
\hline ID & {$[\mathrm{Fe} / \mathrm{H}]$} & $\log (g)$ & $F_{\mathrm{Bol}}$ & $E(B-V)$ & $T_{J}$ & $\Delta T_{J}$ & $T_{H}$ & $\Delta T_{H} T_{K}$ & $\Delta T_{K}$ & $T_{L^{\prime}}$ & $\Delta T_{L^{\prime}}$ & $T_{\text {mean }}$ & $\Delta T_{\text {mean }} n$ \\
\hline SAO111430 & -0.90 & 1.30 & $1.092 \mathrm{E}-09$ & 0.055 & 4251 & 131 & 4243 & 504289 & 32 & 4284 & 152 & 4273 & 523 \\
\hline SAO076423 & -1.23 & 2.00 & $1.907 \mathrm{E}-10$ & 0.070 & 5381 & 116 & 5406 & 765395 & 73 & - & - & 5396 & 853 \\
\hline SAO149407 & -1.64 & 1.20 & $4.424 \mathrm{E}-10$ & 0.001 & 4319 & 109 & 4302 & 474337 & 36 & - & - & 4322 & 412 \\
\hline SAO111667 & -1.94 & 0.80 & $1.452 \mathrm{E}-10$ & 0.100 & 4064 & 181 & 4141 & 574218 & 40 & - & - & 4186 & 472 \\
\hline BS1318 & 0.20 & 2.70 & $4.489 \mathrm{E}-09$ & 0.020 & 4675 & 258 & 4625 & 1364549 & 46 & 4592 & 58 & 4577 & 653 \\
\hline SAO094040 & 0.10 & 2.00 & $2.646 \mathrm{E}-10$ & 0.010 & 5793 & 151 & 5710 & 1085685 & 101 & & - & 5722 & 1163 \\
\hline HR1457 & -0.05 & 1.75 & $3.247 \mathrm{E}-07$ & 0.000 & 3723 & 272 & 3806 & 623856 & 26 & 3888 & 54 & 3866 & 352 \\
\hline SAO149791 & -1.79 & 1.50 & $2.985 \mathrm{E}-10$ & 0.049 & $<3500$ & 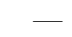 & 3908 & 654020 & 87 & - & - & 4020 & 871 \\
\hline BS1517 & 0.00 & 2.00 & $1.752 \mathrm{E}-09$ & 0.060 & 4553 & 142 & 4560 & 484541 & 39 & _ & - & 4550 & 432 \\
\hline BS1726 & -0.35 & 2.15 & 7.203E-09 & 0.000 & 4071 & 147 & 4170 & 614208 & 40 & - & - & 4193 & 482 \\
\hline BS1805 & -0.18 & 1.60 & $4.708 \mathrm{E}-09$ & 0.020 & 4075 & 203 & 4167 & 484192 & 34 & 4202 & 86 & 4185 & 483 \\
\hline BS1907 & -0.54 & 2.50 & $8.193 \mathrm{E}-09$ & 0.000 & 4643 & 134 & 4667 & 684659 & 49 & 4741 & 44 & 4693 & 523 \\
\hline BS2002 & -0.06 & 2.50 & $4.064 \mathrm{E}-09$ & 0.030 & 4826 & 111 & 4825 & 524784 & 45 & 4892 & 48 & 4833 & \\
\hline BS2012 & -0.10 & 2.25 & $9.515 \mathrm{E}-09$ & 0.000 & 4572 & 122 & 4601 & 484574 & 40 & 4637 & 42 & 4604 & 433 \\
\hline BS2035 & -0.62 & 2.75 & $1.087 \mathrm{E}-08$ & 0.000 & 4583 & 140 & 4586 & 674592 & 44 & 4617 & 55 & 4599 & 543 \\
\hline BS2065 & -0.43 & 0.80 & $4.390 \mathrm{E}-09$ & 0.050 & 3748 & 286 & 3750 & 653873 & 21 & 3906 & 64 & 3881 & 322 \\
\hline HD43039 & -0.31 & 2.50 & $6.492 \mathrm{E}-09$ & 0.000 & 4603 & 183 & 4668 & 774661 & 53 & - & - & 4664 & 632 \\
\hline HD44007 & -1.63 & 2.15 & $2.297 \mathrm{E}-10$ & 0.050 & 4898 & 151 & 4867 & 804840 & 57 & - & - & 4851 & 672 \\
\hline BS2286 & 0.11 & 1.14 & $1.113 \mathrm{E}-07$ & 0.000 & 3597 & 186 & $<3500$ & -3628 & $14:$ & 3644 & 65 & 3631 & 232 \\
\hline BS2319 & 0.00 & 2.00 & $1.256 \mathrm{E}-09$ & 0.025 & 4298 & 149 & 4301 & 454300 & 33 & 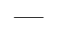 & - & 4300 & 382 \\
\hline HD45282 & -1.40 & 3.30 & $1.952 \mathrm{E}-10$ & 0.020 & 5285 & 152 & 5291 & 229 & 77 & - & - & 5264 & 963 \\
\hline BS2459 & -0.03 & 1.75 & $6.094 \mathrm{E}-09$ & 0.010 & 3769 & 255 & 3975 & 623975 & 87 & - & - & 3975 & 871 \\
\hline BS2557 & 0.30 & 3.40 & $1.206 \mathrm{E}-09$ & 0.030 & 3926 & 201 & 4827 & 944909 & 82 & - & - & 4871 & 882 \\
\hline BS2560 & 0.05 & 3.10 & $5.500 \mathrm{E}-09$ & 0.000 & 5251 & 229 & 5136 & 965062 & 58 & 5048 & 70 & 5092 & 864 \\
\hline BS2649 & 0.00 & 2.00 & 4.707E-09 & 0.030 & 3908 & 510 & 4146 & 2274165 & 70 & - & - & 4161 & 1072 \\
\hline SAO152627 & 0.00 & 2.00 & $5.122 \mathrm{E}-10$ & 0.000 & 3744 & 228 & 3800 & 443856 & 69 & - & - & 3856 & 691 \\
\hline SAO152644 & 0.00 & 2.00 & $5.937 \mathrm{E}-11$ & 0.000 & 6342 & 208 & 6305 & 986317 & 92 & 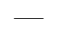 & - & 6317 & 1163 \\
\hline BS2880 & 0.19 & 3.10 & $2.156 \mathrm{E}-09$ & 0.040 & 7432 & 194 & 7409 & 1677441 & $159^{\prime}$ & 7482 & 102 & 7447 & $147 \quad 4$ \\
\hline BS2927 & 0.44 & 3.21 & $2.182 \mathrm{E}-09$ & 0.000 & 6386 & 204 & 6372 & 1876390 & 188 & 6337 & 53 & 6358 & 1164 \\
\hline BS2938 & 0.00 & 1.00 & 7.447E-09 & 0.008 & 3861 & 320 & 3827 & 673822 & 65 & - & - & 3822 & 651 \\
\hline $3 \mathrm{~S} 2985$ & -0.04 & 2.60 & $1.160 \mathrm{E}-08$ & 0.000 & 4983 & 127 & 5026 & 554974 & 55 & 5004 & 58 & 5001 & 563 \\
\hline BS2990 & 0.00 & 2.50 & $1.149 \mathrm{E}-07$ & 0.000 & 4867 & 169 & 4858 & 734819 & 53 & 4904 & 85 & 4854 & 683 \\
\hline BS3003 & -0.27 & 1.67 & $6.955 \mathrm{E}-09$ & 0.000 & 4008 & 254 & 3967 & 753961 & 99 & - & - & 3961 & 991 \\
\hline 3304 & 0.00 & 2.00 & $3.078 \mathrm{E}-09$ & 00 & 3985 & 217 & 4104 & 564096 & 48 & - & - & 4100 & 522 \\
\hline BS3305 & 0.00 & 1.00 & $3.208 \mathrm{E}-09$ & 0.020 & 4059 & 406 & 4001 & 1073994 & 56 & - & - & 3996 & 742 \\
\hline BS3323 & -0.12 & 2.50 & $1.355 \mathrm{E}-08$ & 0.000 & 5125 & 164 & 5172 & 875123 & 58 & 5124 & 94 & 5136 & 884 \\
\hline BS3357 & 0.00 & 1.00 & $5.876 \mathrm{E}-09$ & 0.005 & 3713 & 312 & 3810 & 663803 & 60 & - & - & 3803 & 601 \\
\hline BS3369 & 0.03 & 2.10 & $9.660 \mathrm{E}-10$ & 0.000 & 4686 & 186 & 4759 & 744752 & 53 & 4786 & 78 & 4764 & 663 \\
\hline BS3403 & -0.23 & 2.00 & $5.944 \mathrm{E}-09$ & 0.000 & 4251 & 228 & 4385 & 734388 & 42 & - & - & 4387 & 532 \\
\hline HD73394 & -1.40 & 1.30 & $3.162 \mathrm{E}-10$ & 0.060 & 4662 & 158 & 4559 & 744559 & 50 & 4556 & 55 & 4558 & 583 \\
\hline $\mathrm{S} 3475$ & -0.05 & 2.15 & $7.890 \mathrm{E}-09$ & 0.000 & 4950 & 142 & 4928 & 694886 & 54 & 4925 & 58 & 4911 & 603 \\
\hline BS3547 & -0.04 & 2.50 & $1.858 \mathrm{E}-08$ & 0.000 & 4841 & 169 & 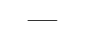 & -4841 & 56 & 4741 & 81 & 4800 & 662 \\
\hline 550 & & 1.00 & $3.489 \mathrm{E}-09$ & & 4439 & 213 & 4288 & 664186 & 57 & . & - & 4233 & 612 \\
\hline BS3660 & 0.00 & 1.00 & $5.414 \mathrm{E}-09$ & 0.000 & 4127 & 195 & 3891 & 393876 & 40 & - & - & 3876 & 401 \\
\hline BS3664 & -0.85 & 2.20 & $1.326 \mathrm{E}-09$ & 0.015 & 5049 & 136 & 5013 & 655033 & 54 & 5087 & 43 & 5050 & 624 \\
\hline & 00 & 1.53 & & ח & & 248 & 3781 & 443854 & $25:$ & 3900 & 131 & 3861 & 422 \\
\hline HD81192 & -0.64 & 2.60 & $8.846 \mathrm{E}-10$ & 0.010 & 4698 & 107 & 4687 & 564701 & 49 & 4775 & 141 & 4707 & 663 \\
\hline & -1.85 & 2.75 & 5.3 & & & 134 & 5991 & 946013 & 93 & - & - & 6005 & 1043 \\
\hline 12 & -1.47 & 1.25 & 2.0 & 0 & 4 & 130 & 4431 & 554473 & 42 & - & - & 4455 & 482 \\
\hline BS3905 & 0.16 & 2.40 & $1.082 \mathrm{E}-08$ & 0.000 & 4401 & 202 & 4529 & 754492 & 51 & 4483 & 75 & 4500 & 653 \\
\hline HD 87140 & -1.86 & 2.65 & $7.949 \mathrm{E}-11$ & 00 & 4933 & 164 & 5012 & 915034 & 70 & - & - & 5024 & 793 \\
\hline BS3994 & 0.10 & 2.83 & $1.158 \mathrm{E}-08$ & 0.000 & 4850 & 161 & 4918 & 834869 & 61 & 4810 & 80 & 4865 & 733 \\
\hline & -2.60 & 1.30 & & & 46 & 153 & 4582 & 964612 & 60 & - & 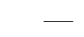 & 4600 & 742 \\
\hline BS4030 & -0.03 & 4.00 & $1.104 \mathrm{E}-09$ & 0.000 & 5604 & 129 & 5641 & 805657 & 95 & - & - & 5637 & 973 \\
\hline BS4042 & & 3.76 & $1.961 \mathrm{E}-09$ & 0.000 & 6976 & 184 & 6981 & 1437012 & $137^{\prime}$ & 7102 & 65 & 7040 & 1144 \\
\hline HD093529 & -1.70 & 2.00 & $7.705 \mathrm{E}-11$ & 0.065 & 4850 & 98 & 4812 & 624835 & 52 & - & - & 4825 & 572 \\
\hline BS4246 & -0.10 & 2.30 & $4.304 \mathrm{E}-09$ & 0.000 & 4270 & 216 & 4250 & 704222 & 40 & 4227 & 66 & 4231 & 553 \\
\hline BS4247 & -0.20 & 2.80 & $1.051 \mathrm{E}-08$ & & 4614 & 123 & 4648 & 594636 & 47 & 4646 & 57 & 4643 & 543 \\
\hline BS4291 & -0.18 & 2.75 & $4.510 \mathrm{E}-09$ & 0.000 & 4468 & 193 & 4497 & 724486 & 46 & 4491 & 72 & 4490 & 613 \\
\hline BS4309 & 0.00 & 3.00 & 1.007E-09 & 0.010 & 7832 & 161 & 7816 & 1477820 & 134 & - & - & 7822 & 1473 \\
\hline
\end{tabular}


Table 6. continued

\begin{tabular}{|c|c|c|c|c|c|c|c|c|c|c|c|c|}
\hline$\overline{I D}$ & {$[\mathrm{Fe} / \mathrm{H}]$} & $\log (g)$ & $\overline{F_{\mathrm{Bol}}}$ & $E(B-V)$ & $\overline{T_{J}}$ & $\Delta T_{J} T_{H}$ & $\Delta T_{H} T_{K}$ & $\Delta T_{K}$ & $T_{L^{\prime}}$ & $\Delta T_{L^{\prime}}$ & $T_{\text {mean }}$ & $\Delta T_{\text {mean }} n$ \\
\hline$\overline{B S 4336}$ & 0.00 & 2.00 & $5.330 \mathrm{E}-09$ & 0.000 & $<3500$ & -3568 & 623655 & 78 & 3646 & 65 & 3650 & 702 \\
\hline BS4382 & -0.40 & 2.50 & $1.450 \mathrm{E}-08$ & 0.000 & 4476 & $117-$ & -4504 & 39 & 4423 & 48 & 4468 & 432 \\
\hline BS4392 & -0.03 & 2.30 & $3.238 \mathrm{E}-09$ & 0.000 & 4915 & 1224905 & 1164854 & 50 & 4880 & 88 & 4872 & 753 \\
\hline $\mathrm{BD}+042466$ & -1.88 & 1.80 & $2.013 \mathrm{E}-11$ & 0.016 & 5067 & 1515094 & 745123 & 58 & - & 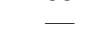 & 5103 & 803 \\
\hline BS4432 & -0.39 & 1.67 & 7.972E-09 & 0.000 & 3629 & 1873792 & 713920 & 58 & 3876 & 32 & 3891 & 412 \\
\hline BS4452 & -0.40 & 2.50 & $1.019 \mathrm{E}-09$ & 0.020 & 4670 & 1214680 & 624687 & 48 & 4737 & 51 & 4702 & \\
\hline BS4474 & -0.33 & 2.00 & $1.226 \mathrm{E}-09$ & 0.030 & 4885 & 1134883 & 574883 & 50 & 4827 & 138 & 4874 & 673 \\
\hline $\mathrm{BD}+222411$ & -1.95 & 1.25 & 4.144E-11 & 0.018 & 4354 & 1864346 & 774388 & 47 & - & - & 4372 & 582 \\
\hline BD-01 2582 & -2.20 & 1.20 & $4.581 \mathrm{E}-11$ & 0.005 & 5177 & 1635151 & 955134 & 71 & . & - & 5148 & 983 \\
\hline BS4518 & -0.50 & 1.60 & $1.355 \mathrm{E}-08$ & 0.000 & 4339 & 117 & -4374 & 36 & 4319 & 40 & 4348 & 382 \\
\hline HD103545 & -2.30 & 2.00 & $6.137 \mathrm{E}-11$ & 0.000 & 4655 & 1564659 & 894671 & 59 & - & - & 4666 & 712 \\
\hline $\mathrm{BD}+092574$ & -2.40 & 2.00 & $2.077 \mathrm{E}-11$ & 0.000 & 4855 & 1454825 & 824875 & 63 & - & - & 4853 & 712 \\
\hline BS4608 & -0.38 & 2.75 & 7.399E-09 & 0.000 & 4789 & 1054834 & 534831 & 48 & 4807 & 51 & 4824 & \\
\hline HD105546 & -1.40 & 2.40 & $1.093 \mathrm{E}-10$ & 0.000 & 5227 & 1535203 & 915165 & 66 & - & - & 5190 & 923 \\
\hline HD106373 & -2.48 & 2.80 & $9.294 \mathrm{E}-11$ & 0.060 & 6088 & 1866076 & 1146111 & 108 & - & - & 6093 & 1283 \\
\hline BS4695 & -0.40 & 2.40 & 4.297E-09 & 0.000 & 4328 & 1124364 & 464377 & 37 & 4364 & 43 & 4369 & 423 \\
\hline HD107328 & -0.47 & 2.20 & $4.274 \mathrm{E}-09$ & 0.000 & 4317 & 2084418 & 764384 & 43 & & . & 4396 & 552 \\
\hline BS4716 & -0.25 & 2.30 & $3.684 \mathrm{E}-09$ & 0.000 & 5017 & 1135053 & 605033 & 59 & 5010 & 106 & 5033 & 774 \\
\hline HD108317 & -2.30 & 2.40 & $1.891 \mathrm{E}-10$ & 0.000 & 5220 & 1175248 & 785230 & 75 & - & - & 5234 & 863 \\
\hline HD108577 & -2.50 & 2.00 & $4.936 \mathrm{E}-11$ & 0.020 & 4987 & 1135008 & 755031 & 64 & - & - & 5020 & 692 \\
\hline HD110184 & -2.26 & 0.90 & $2.157 \mathrm{E}-10$ & 0.000 & 4187 & 1554213 & 644273 & 39 & - & - & 4250 & 482 \\
\hline HD110281 & 0.00 & 2.00 & $1.170 \mathrm{E}-10$ & 0.015 & 3668 & 2043890 & 723950 & 54 & - & - & 3950 & 541 \\
\hline BS4883 & 0.11 & 2.50 & $2.869 \mathrm{E}-09$ & 0.000 & 5597 & 875567 & 865581 & 95 & 5565 & 50 & 5589 & 764 \\
\hline $\mathrm{BD}+73566$ & -1.32 & 2.00 & $5.413 \mathrm{E}-11$ & 0.000 & 5642 & 1615616 & 965626 & 90 & & 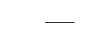 & 5626 & 1083 \\
\hline BS4902 & 0.00 & 2.00 & $1.754 \mathrm{E}-08$ & 0.020 & 3638 & 2143510 & 663630 & 54 & 3586 & 50 & 3607 & 522 \\
\hline $\mathrm{BD}+102495$ & -1.83 & 2.00 & $4.184 \mathrm{E}-11$ & 0.003 & 4946 & 1194916 & 694964 & 74 & & $\ldots$ & 4939 & 712 \\
\hline BS4932 & 0.02 & 2.75 & $2.251 \mathrm{E}-08$ & 0.000 & 5089 & 1035067 & 555031 & 54 & 5004 & 61 & 5043 & 644 \\
\hline BS4981 & -0.15 & 4.00 & $2.465 \mathrm{E}-09$ & 0.000 & 6259 & 1336250 & 1116255 & 95 & 6182 & 145 & 6240 & 1184 \\
\hline HD115444 & -2.70 & 1.85 & $9.165 \mathrm{E}-11$ & 0.000 & 4731 & 1024703 & 714735 & 53 & 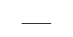 & - & 4721 & 612 \\
\hline BS5017 & 0.30 & 3.75 & 3.091E-09 & 0.000 & 7117 & 1877157 & 1707178 & 160 & 7096 & 217 & 7141 & 1814 \\
\hline AO028774 & 0.00 & 2.00 & $3.738 \mathrm{E}-10$ & 0.020 & 4648 & 2304575 & 634584 & 45 & - & - & 4580 & 522 \\
\hline BS5102 & -0.50 & 2.25 & $1.317 \mathrm{E}-09$ & 0.020 & 4706 & 1354720 & 604712 & 47 & 4683 & 64 & 4706 & 563 \\
\hline $\mathrm{BD}+182757$ & -2.60 & 2.00 & $4.070 \mathrm{E}-11$ & 0.000 & 4823 & 944841 & 644864 & 56 & - & 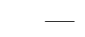 & 4853 & 602 \\
\hline HD119516 & -2.50 & 2.50 & $6.983 \mathrm{E}-11$ & 0.000 & 5448 & 1285487 & 865483 & 83 & - & - & 5476 & \\
\hline HD121135 & -1.70 & 2.00 & $6.025 \mathrm{E}-11$ & 0.011 & 4942 & 1034927 & 674940 & 60 & - & 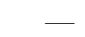 & 4934 & 632 \\
\hline BS5263 & 0.00 & 3.00 & $8.246 \mathrm{E}-10$ & 0.015 & 7663 & 1867676 & 1597673 & 148 & - & - & 7671 & 1633 \\
\hline HD122563 & -2.62 & 1.60 & $1.248 \mathrm{E}-09$ & 0.000 & 4542 & 964534 & 854589 & 55 & 4578 & 51 & 4572 & 613 \\
\hline SAO063927 & 0.00 & 2.00 & $3.435 \mathrm{E}-11$ & 0.000 & 5772 & 1325898 & 895917 & 86 & - & 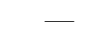 & 5867 & 993 \\
\hline SAO158392 & 0.00 & 2.50 & $6.437 \mathrm{E}-11$ & 0.020 & 5940 & 1315898 & 895909 & 89 & - & - & 5913 & 1003 \\
\hline BS5301 & 0.00 & 0.93 & $1.217 \mathrm{E}-08$ & 0.000 & $<3500$ & 3500 & 493669 & 67 & 3679 & 108 & 3672 & 82 \\
\hline HD124358 & -2.20 & 1.75 & $6.484 \mathrm{E}-11$ & 0.042 & 4718 & 1004668 & 714703 & 55 & - & 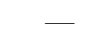 & 4688 & 622 \\
\hline $\mathrm{BD}+092860$ & -2.60 & 2.00 & $1.470 \mathrm{E}-11$ & 0.004 & 5391 & 2815278 & 785260 & 83 & - & - & 5285 & 1063 \\
\hline HR5340 & -0.50 & 1.75 & $4.830 \mathrm{E}-07$ & 0.000 & 4187 & 2254166 & 794247 & 38 & 4263 & 64 & 4233 & 553 \\
\hline $\mathrm{BD}+082856$ & -2.05 & 1.00 & $3.934 \mathrm{E}-11$ & 0.005 & 4541 & 944506 & 544520 & 43 & - & - & 4514 & 482 \\
\hline HD126587 & -2.70 & 2.00 & $9.370 \mathrm{E}-11$ & 0.070 & 4790 & 924776 & 654809 & 52 & - & - & 4794 & 582 \\
\hline HD126778 & 0.11 & 2.00 & 1.815 & 0.000 & 4859 & 1534779 & 804778 & 56 & - & - & 4778 & 662 \\
\hline $\mathrm{BD}+182890$ & -1.61 & 2.00 & $3.954 \mathrm{E}-11$ & 0.030 & 5063 & 1085049 & 85060 & 61 & - & - & 5057 & 743 \\
\hline BS5429 & -0.30 & 1.80 & 1.638 & 0.000 & 4307 & 1754267 & 714275 & 34 & 4267 & 77 & 4271 & 533 \\
\hline HD128279 & -2.22 & 3.00 & $2.185 \mathrm{E}-10$ & 0.050 & 5274 & 1575282 & 945305 & 82 & - & 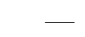 & 5290 & 1033 \\
\hline BS5480 & -0.22 & 2.00 & 3.729E-09 & 0.000 & 4872 & 1064880 & 544831 & 47 & 4854 & 87 & 4854 & 583 \\
\hline BS5535 & -0.38 & 2.65 & $3.657 \mathrm{E}-09$ & 0.000 & 4672 & 1194720 & 564713 & 48 & 4709 & 93 & 4715 & 613 \\
\hline HD130952 & -0.38 & 2.50 & $3.761 \mathrm{E}-09$ & 0.010 & 4623 & 1784702 & 784713 & 55 & - & — & 4708 & 652 \\
\hline $\mathrm{BD}+302611$ & -1.40 & 1.00 & $1.048 \mathrm{E}-10$ & 0.1 & 4258 & 2094141 & 784244 & 40 & - & - & 4209 & 532 \\
\hline HD135722 & -0.43 & 2.60 & $1.361 \mathrm{E}-08$ & 0.000 & 4644 & 1774776 & 724763 & 52 & - & - & 4768 & 602 \\
\hline BS5681 & -0.43 & 2.50 & $1.342 \mathrm{E}-08$ & 0.000 & 4778 & 103 & -4794 & 49 & 4802 & 49 & 4798 & 492 \\
\hline BS5694 & -0.13 & 3.95 & $2.455 \mathrm{E}-09$ & 0.000 & 6058 & 1246067 & 866050 & 89 & 5964 & 104 & 6036 & 994 \\
\hline BS5709 & -0.38 & 2.70 & $2.194 \mathrm{E}-09$ & 0.000 & 4627 & 1774692 & 564680 & 48 & 4624 & 170 & 4677 & 673 \\
\hline 787 & -0.44 & 2.80 & $9.706 \mathrm{E}-09$ & 0.010 & 4651 & 1384732 & 554717 & 47 & 4686 & 47 & 4711 & 493 \\
\hline BS5802 & -0.13 & 3.00 & $2.474 \mathrm{E}-09$ & 0.000 & 4946 & 1244974 & 624950 & 56 & 4953 & 54 & 4958 & 573 \\
\hline BS5804 & 0.00 & 2.50 & $1.053 \mathrm{E}-09$ & 0.000 & 6661 & 1226670 & 1056655 & 102 & - & - & 6662 & 1093 \\
\hline
\end{tabular}


Table 6. continued

\begin{tabular}{|c|c|c|c|c|c|c|c|c|c|c|c|c|}
\hline ID & $\mathrm{H}]$ & $\overline{\log (g)}$ & $\overline{E_{\mathrm{P}}+1}$ & $\overline{B-V)}$ & $\overline{T_{J}}$ & $\Delta T_{J} T_{H}$ & $\Delta T_{H} T_{K}$ & $\Delta T_{K}$ & $T T_{L^{\prime}}$ & $\Delta T_{L^{\prime}}$ & $T_{\text {mean }}$ & $\Delta T_{\text {mean }} n$ \\
\hline$\overline{B S 5823}$ & -0.55 & 3.20 & $2.581 \mathrm{E}-09$ & 0.013 & 4889 & 1184895 & 564890 & 52 & 4893 & 88 & 4893 & 623 \\
\hline BS5824 & -0.10 & 2.30 & $5.166 \mathrm{E}-09$ & 0.070 & 4334 & 1304410 & 524403 & 40 & 4317 & 123 & 4392 & 573 \\
\hline HD14153 & -1.57 & 1.00 & $9.446 \mathrm{E}-11$ & 016 & 4874 & 1444503 & 724433 & 49 & & & 4461 & 58 \\
\hline BS5889 & -0.32 & 3.10 & $4.200 \mathrm{E}-09$ & 000 & 5143 & 1185187 & 705138 & 52 & 5252 & 69 & 5180 & 71 \\
\hline $\mathrm{BD}+053098$ & -2.60 & 2.00 & $2.284 \mathrm{E}-11$ & 038 & 4840 & 944866 & 684893 & 58 & - & - & 4881 & 632 \\
\hline $\mathrm{BD}+112998^{a}$ & 1.15 & 2.50 & $275 \mathrm{E}-11$ & 048 & 9098 & 2177191 & 1406966 & 128 & - & - & 7073 & 1342 \\
\hline $\mathrm{BD}+093223$ & -2.30 & 2.00 & $7.166 \mathrm{E}-11$ & 060 & 5395 & 1225366 & 795342 & 73 & - & — & 5363 & 87 \\
\hline BS6189 & -0.62 & 3.95 & $7.891 \mathrm{E}-10$ & 010 & 6008 & 1356014 & 866016 & 87 & - & - & 6013 & 98 \\
\hline 213 & 0.00 & 3.00 & $1.058 \mathrm{E}-09$ & 000 & 6445 & 1326449 & 1016466 & 104 & 6519 & 84 & 6474 & 103 \\
\hline BS6220 & -0.20 & 3.00 & $1.261 \mathrm{E}-08$ & 0.000 & 4917 & 1304966 & 834931 & 55 & 4933 & 93 & 4942 & 73 \\
\hline 0151937 & 0.00 & 2.00 & 079E-09 & 010 & 4268 & 2314322 & 644254 & 47 & - & - & 4283 & 54 \\
\hline 66279 & 0.00 & 3.00 & $801 \mathrm{E}-09$ & 00 & 6893 & 1286878 & 1116888 & 105 & - & - & 6886 & 114 \\
\hline 394 & -0.14 & 3.95 & $793 \mathrm{E}-10$ & 010 & 6159 & 1286169 & 896156 & 93 & - & - & 6162 & 101 \\
\hline 469 & 0.00 & 2.00 & $733 \mathrm{E}-09$ & 000 & 5398 & 1575314 & 815263 & 74 & - & - & 5309 & 93 \\
\hline 36480 & 0.00 & 2.00 & $1.251 \mathrm{E}-09$ & 000 & 7392 & 1547399 & 1407399 & 131 & - & - & 7397 & 141 \\
\hline $\mathrm{BD}+17$ & -2.04 & 1.80 & E-11 & & 5272 & 1095235 & 755213 & 71 & - & - & 5236 & 82 \\
\hline BS6541 & -0.24 & 3.95 & $1.415 \mathrm{E}-09$ & 00 & 6090 & 1616112 & 1036084 & 98 & - & - & 6096 & 115 \\
\hline 594 & -0.13 & 4.00 & -09 & 00 & 6496 & 1706500 & 1166472 & 113 & - & - & 6488 & 128 \\
\hline 503 & 0.05 & 2.30 & $952 \mathrm{E}-08$ & 000 & 4497 & 1514546 & 554524 & 40 & - & - & 4533 & 46 \\
\hline HD161770 & -2.12 & 2.50 & $5.004 \mathrm{E}-11$ & 0.075 & 5291 & 1575275 & 945278 & 80 & - & - & 5280 & 102 \\
\hline & 04 & 30 & & & 4457 & 240 & 20 & 74 & - & 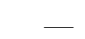 & 4518 & \\
\hline 688 & -0.12 & 2.50 & -08 & 00 & 4467 & 1444487 & 564472 & 42 & 4457 & 55 & 4472 & 50 \\
\hline 698 & 0.09 & 2.60 & -08 & 00 & 4868 & 1354900 & 714852 & 5 & 4865 & 88 & 4871 & 69 \\
\hline & -0.25 & 1.50 & -08 & & 3758 & 2833862 & 723955 & 33 & 3898 & 56 & 3934 & 42 \\
\hline & 0.00 & 2.00 & $683 \mathrm{E}-10$ & 00 & 5533 & 1075490 & 735460 & 72 & - & - & 5490 & 81 \\
\hline $\mathrm{H}$ & -2.20 & 1.50 & $960 \mathrm{E}-10$ & 130 & 4084 & 1344153 & 644251 & 50 & 4350 & 108 & 4237 & 67 \\
\hline & 0.15 & 2.95 & 09 & & 4925 & 1635003 & 634966 & 55 & 4937 & 69 & 4969 & 62 \\
\hline $\mathrm{S}$ & -1.21 & .31 & & & 4878 & 1064934 & 665008 & 56 & - & - & 4974 & 61 \\
\hline $\mathrm{B}$ & 0.06 & 65 & & & 5006 & 1085003 & 654952 & 62 & 5025 & 70 & 4994 & 73 \\
\hline BS6795 & 00 & 2.00 & $1.302 \mathrm{E}-09$ & 010 & 6697 & 1636660 & 216636 & 115 & - & 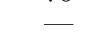 & 6661 & 303 \\
\hline & 0.22 & U & & & & 12 & 86 & 105 & - & - & 6312 & 121 \\
\hline & 00 & 00 & 09 & & 4959 & 174939 & 624886 & 52 & 4956 & 138 & 4918 & 70 \\
\hline & & 0 & & & & 95736 & & 79 & - & - & 5745 & \\
\hline & .30 & 0 & & & & 94 & 644 & 0 & 4861 & 84 & 4785 & 63 \\
\hline & .19 & 3.10 & & & & c & 554 & 48 & 484 & 60 & 4835 & 54 \\
\hline & & & & & & 4 & 644 & 41 & 4409 & 46 & 4428 & 49 \\
\hline & 91 & 0 & & & 34 & 95060 & 825062 & 69 & - & - & 5075 & 91 \\
\hline & & 0 & & & & 27115 & 1267121 & 117 & - & - & 7120 & 128 \\
\hline & .07 & & & & & 1294990 & 614 & 55 & 4864 & 41 & 4928 & 51 \\
\hline BS6973 & 0.09 & 2.10 & $1.324 \mathrm{E}-08$ & 000 & 4233 & 2704254 & 904266 & 50 & 4218 & 69 & 4248 & 66 \\
\hline & .12 & & & & & & & 43 & - & - & 4485 & 51 \\
\hline & & 00 & & & 6613 & 56648 & 1176 & 107 & - & - & 6655 & 129 \\
\hline & & & & & & 8 & 833 & 54 & - & - & & 54 \\
\hline & & & & & 6 & 03 & 828230 & 170 & - & - & 8210 & 178 \\
\hline & & & & & & 38 & 975 & 92 & - & - & 5676 & 110 \\
\hline & & & & & & 542 & & & - & 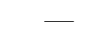 & 4283 & \\
\hline & -1 & & & & & 28 & & 66 & - & - & 5041 & 90 \\
\hline & & & & & & 6 & 64 & (1) & 48 & 59 & & 57 \\
\hline & & & & & & 2 & 804 & o & 4924 & 70 & 4840 & 72 \\
\hline BS7317 & -0.00 & 1.40 & $2.490 \mathrm{E}-09$ & 0.040 & 3805 & 1973892 & 663989 & 34 & 4038 & 55 & 4008 & 42 \\
\hline & & & & & & 8 & 916 & 96 & 64 & 132 & 6285 & 111 \\
\hline & & 2. & & & & 1294 & & 54 & 4968 & 68 & 4947 & 61 \\
\hline & & & & & & 0 & 694 & 52 & - & - & 4776 & - \\
\hline B & -2.30 & 2.5 & & & & 1826378 & 1256413 & 122 & - & - & 6419 & 138 \\
\hline & -157 & 1.8 & & & & 1255 & & 75 & - & - & 5587 & 7 \\
\hline & & & & & & 4 & 454 & 43 & 4393 & 139 & 4473 & 7 \\
\hline & & 2. & & & & 1944 & 674 & 45 & - & - & 4555 & 542 \\
\hline & -2. & 0. & & & & 1 & 804 & 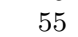 & - & - & 4598 & 65 \\
\hline SAO16 & -1.87 & 0.7 & $4.979 \mathrm{E}-10$ & & 4357 & 1814229 & 804292 & 41 & - & - & 4271 & 54 \\
\hline BS7569 & 0.06 & 2.00 & $9.517 \mathrm{E}-10$ & 0.000 & 5699 & 1185637 & 895623 & 80 & - & - & 5648 & 93 \\
\hline
\end{tabular}


Table 6. continued

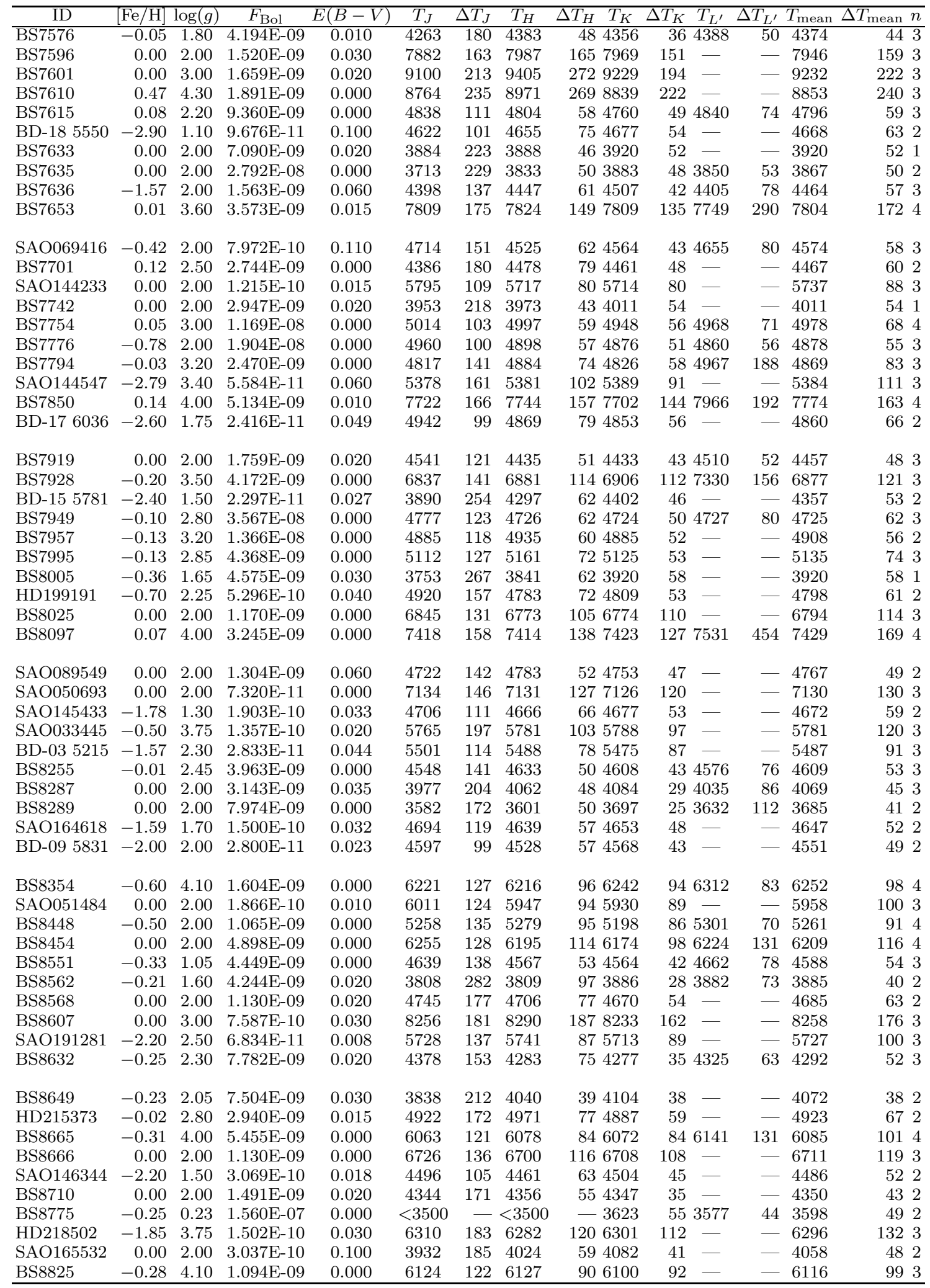


Table 6. continued

\begin{tabular}{|c|c|c|c|c|c|c|c|c|c|c|c|c|c|}
\hline ID & {$[\mathrm{Fe} / \mathrm{H}]$} & $\log (g)$ & $F_{\text {Bol }}$ & $E(B-V)$ & $\overline{T_{J}}$ & $\Delta T_{J}$ & $T_{H}$ & $\Delta T_{H} \quad T_{K}$ & $\Delta T_{K}$ & $T_{L^{\prime}}$ & $\Delta T_{L^{\prime}}$ & $T_{\text {mean }}$ & $\Delta T_{\text {mean }} n$ \\
\hline$\overline{\mathrm{BS} 8834}$ & 0.00 & 2.00 & $1.796 \mathrm{E}-08$ & 0.000 & 3498 & 164 & 3688 & 793762 & 51 & - & - & 3762 & 511 \\
\hline HD219615 & -0.44 & 2.50 & $1.102 \mathrm{E}-08$ & 0.000 & 4837 & 159 & 4828 & 724824 & 55 & - & - & 4826 & 622 \\
\hline BS8878 & 0.00 & 2.00 & 4.519E-09 & 0.000 & 4304 & 226 & 4228 & 734238 & 57 & - & - & 4234 & 642 \\
\hline BS8905 & 0.11 & 2.00 & $4.548 \mathrm{E}-09$ & 0.000 & 5904 & 122 & 5853 & 975834 & 91 & 6201 & 137 & 5928 & 1094 \\
\hline SAO191895 & -1.59 & 1.80 & $3.669 \mathrm{E}-11$ & 0.008 & 4462 & 112 & 4449 & 614478 & 43 & 一 & - & 4466 & 502 \\
\hline BS8916 & -0.10 & 2.40 & $6.691 \mathrm{E}-09$ & 0.000 & 4720 & 138 & 4714 & 634672 & 46 & 4727 & 86 & 4699 & 613 \\
\hline BS8923 & -0.03 & 2.91 & $4.768 \mathrm{E}-09$ & 0.000 & 5007 & 133 & 4998 & 614936 & 54 & 4943 & 95 & 4967 & 764 \\
\hline HD221170 & -2.16 & 1.10 & $4.027 \mathrm{E}-10$ & 0.060 & 4520 & 134 & 4416 & 804434 & 53 & 4332 & 150 & 4410 & 793 \\
\hline 8930 & 0.00 & 2.00 & 2.892E-09 & 0.000 & 4697 & 130 & 4695 & 784646 & 51 & 4546 & 42 & 4615 & 533 \\
\hline BS8961 & -0.60 & 2.80 & $1.089 \mathrm{E}-08$ & 0.000 & 4560 & 105 & 4620 & 654589 & 46 & 4534 & 49 & 4578 & 523 \\
\hline BS9057 & 0.00 & 2.00 & 1.306E-09 & 0.050 & 5613 & 111 & 5511 & 755471 & 72 & 5518 & 53 & 5520 & 734 \\
\hline SAO105082 & -1.10 & 0.50 & $5.782 \mathrm{E}-10$ & 0.160 & $<3500$ & - & $<3500$ & -3654 & 70 & $<3500$ & - & 3654 & 701 \\
\hline $\mathrm{BD}+541323$ & -1.40 & 2.30 & $5.657 \mathrm{E}-11$ & 0.005 & 5186 & 116 & 5191 & 745182 & 68 & 一 & - & 5186 & 813 \\
\hline $\mathrm{BD}+521601$ & -1.50 & 2.05 & $1.037 \mathrm{E}-10$ & 0.020 & 4924 & 97 & 4906 & 614915 & 54 & - & - & 4911 & 572 \\
\hline $\mathrm{SAO} 024707$ & 0.00 & 2.00 & $1.459 \mathrm{E}-10$ & 0.000 & 4903 & 173 & 4964 & 784939 & 62 & - & - & 4950 & 692 \\
\hline $\mathrm{BD}+581218$ & -2.63 & 1.90 & $3.392 \mathrm{E}-11$ & 0.000 & 4968 & 123 & 4984 & 854985 & 65 & - & - & 4985 & 742 \\
\hline SAO078681 & 0.00 & 2.00 & $3.883 \mathrm{E}-11$ & 0.000 & 5718 & 165 & 6146 & 1106280 & 101 & — & - & 6095 & 1203 \\
\hline HD268518 & 0.11 & 2.00 & $2.879 \mathrm{E}-10$ & 0.060 & 5746 & 160 & 6035 & 1105869 & 103 & - & - & 5899 & 1203 \\
\hline SAO103301 & 0.00 & 2.00 & $1.642 \mathrm{E}-10$ & 0.000 & 4802 & 124 & 4824 & 504780 & 45 & - & - & 4801 & 472 \\
\hline
\end{tabular}

${ }^{a} \mathrm{BD}+112998$ probable misidentification in the program of near IR photometry.

${ }^{b}$ HD 268518 variable?

and consequently extinction corrections have to be applied. For this purpose, we have estimated $E(B-V)$ for each star in the sample in order to correct both the bolometric and monochromatic fluxes. Where the values of the estimated extinction were considered significant, the colours have been corrected according to the extinction law $\left(A_{\lambda}=f\left(A_{V}, \lambda\right)\right)$ compiled by Landolt-Börnstein (1982). Two independent methods have been considered for assigning $E(B-V)$ to field stars. The first one is based on the work by Anthony-Twarog \& Twarog (1994), which provide $E(b-y)$ obtained with the reddening maps of Burnstein \& Heiles (1982). In this case, we have considered $E(B-V)=1.37 E(b-y)$ (Crawford 1975). The second one makes use of the extinction models for the galaxy compiled by Hakkila et al. (1997) and the distances calculated from Hipparcos parallaxes. The values obtained by Anthony-Twarog \& Twarog (1994) are preferred for metal-poor giants, since the parallaxes of these stars are affected by errors which made the second method more uncertain.

As for the stars of globular clusters, we have adopted an average of the most reliable values quoted in literature, and, except for M 71, we have restricted our analysis to low reddening clusters.

In Tables 6 and 7 we present the reddening correction applied to the stars of the sample. It is worth noticing that a third of the field stars of the sample needed a reddening correction $E(B-V) \geq 0.02 \mathrm{mag}$, and that $\sim 10 \%$ of them have $E(B-V) \geq 0.05$ mag.

The change in temperature induced by $E(B-V)=$ $0.05 \mathrm{mag}$ when applying the IRFM varies from $2.1 \%$ at $3500 \mathrm{~K}$ to $4.5 \%$ at $7500 \mathrm{~K}$. The hotter the star the stronger the effect since the proportion of flux radiated in the visible/UV wavelength range is greater.

\subsection{Metallicity and surface gravity}

The effective temperature determination by means of Eq. (3) requires an estimate of the stellar metallicity and surface gravity. These parameters, however, need not be very accurate as mentioned in Sect. 3.1. In particular, it may be concluded that 0.5 dex and 0.3 dex uncertainties in $\log (g)$ and $[\mathrm{Fe} / \mathrm{H}]$, respectively, are sufficient to obtain temperatures to an accuracy of $1-2 \%$ (see Fig. 2). Therefore, as far as the surface gravity is concerned, it is enough to consider an average surface gravity according to the spectral type, although spectroscopic determinations of surface gravities have been adopted when available from literature.

A number of stars in the sample had their metal abundance determined from fine spectroscopic analysis included in the Catalogue of $[\mathrm{Fe} / \mathrm{H}]$ determinations of Cayrel de Strobel et al. (1997) with a mean accuracy within 0.15 dex. We have preferred these determinations, but for stars lacking spectroscopic analysis, photometric metallicity calibrations based on Strömgren photometry (Anthony-Twarog \& Twarog 1994), and on $\delta_{0.6}(U-B)$ index (Carney 1979), with accuracies oscillating between $0.20-030$ dex, were used.

The adopted gravities and metallicities are listed in Tables 6 and 7 . 

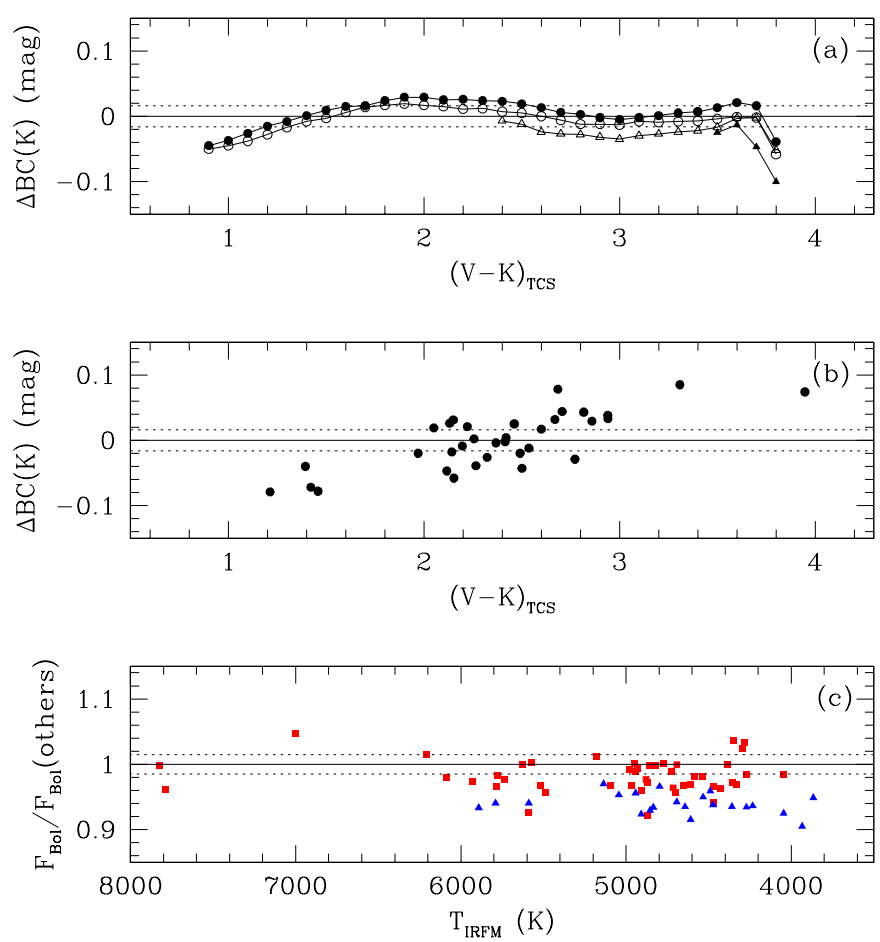

Fig. 4. a) Difference between the bolometric correction to $K_{\mathrm{TCS}}$ obtained here and those theoretically derived by Bessell et al. (1998): Solid circles: ATLAS9 Kurucz (1995) overshooting models, open circles: ATLAS9 Kurucz (1995) no overshooting models, solid triangles; NMARCS giant branch models of Plez et al. (1992), open triangles: NMARCS giant branch models of Plez (1995) b) Difference between the bolometric correction to $K_{\mathrm{TCS}}$ obtained here and that of Flower (1996); c) Ratios of the fluxes presented in this work to those obtained by Bell \& Gustafsson (1989) (triangles), and Blackwell \& Lynas-Gray (1998) (squares). Dotted lines show the internal error of our calibration $(1.5 \%)$

\section{The determination of temperatures}

According to the procedure described in the preceding sections, we have derived three (four) effective temperatures for each star in the sample by applying the IRFM at the IR wavelengths considered (Eq. 3). The individual values of $T_{J}, T_{H}, T_{K}\left(\right.$ and $\left.T_{L^{\prime}}\right)$ derived with their corresponding errors, are listed in Tables 6 and 7.

The final temperature was derived as an average of $T_{J}, T_{H}, T_{K}$ (and $\left.T_{L^{\prime}}\right)$ weighted with the inverse of their errors:

$$
\overline{T_{\mathrm{IRFM}}}=\frac{\sum_{i=J, H, K, L} \frac{T_{i}}{\Delta T_{i}}}{\sum_{i=J, H, K, L} \frac{1}{\Delta T_{i}}} .
$$

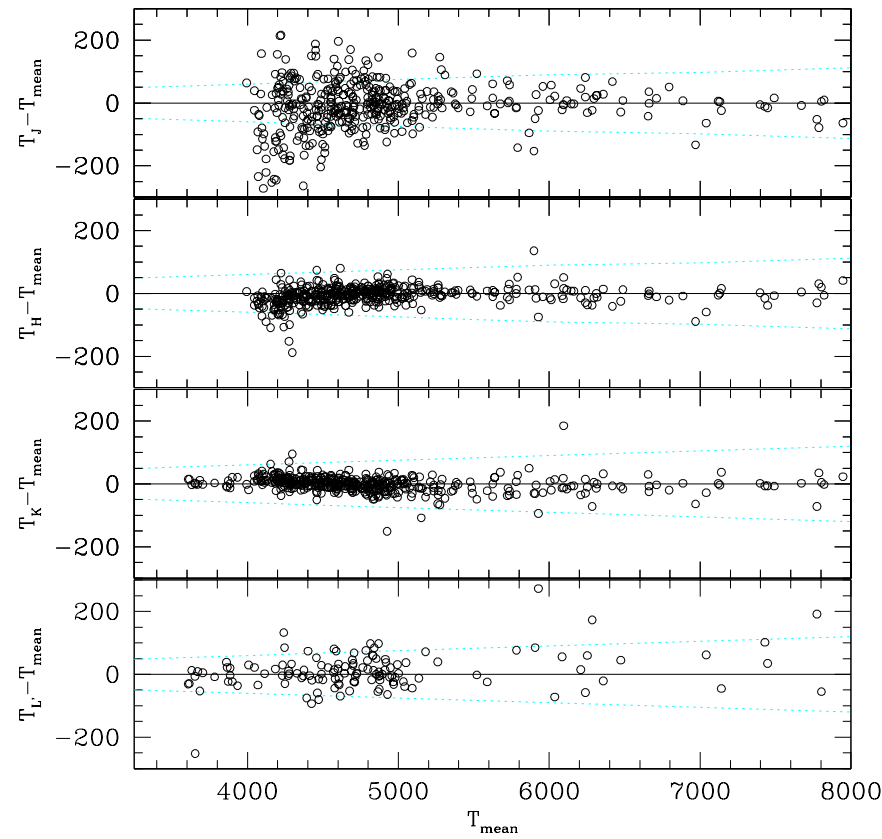

Fig. 5. Differences $T_{J}-T_{\text {mean }}, T_{H}-T_{\text {mean }}, T_{K}-T_{\text {mean }}$ and $T_{L^{\prime}}-T_{\text {mean }}$ versus effective temperature (only temperatures obtained from more than one filter are displayed). The dotted lines show the region $|\Delta T| \leq 1.5 \%$

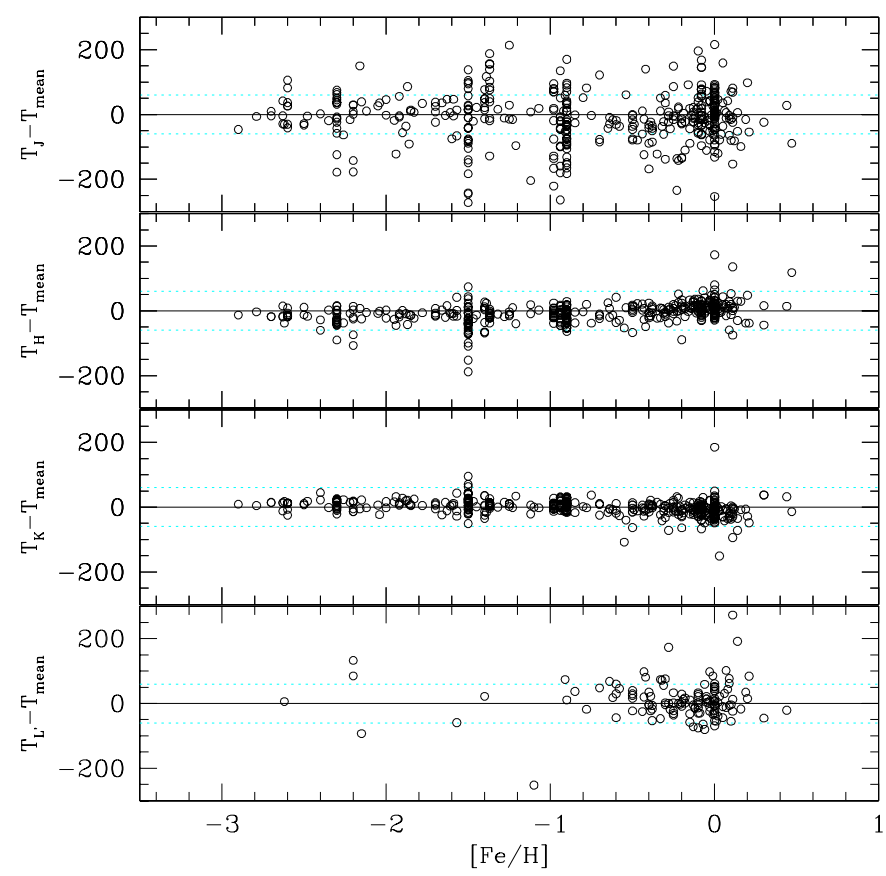

Fig. 6. Differences $T_{J}-T_{\text {mean }}, T_{H}-T_{\text {mean }}, T_{K}-T_{\text {mean }}$ and $T_{L^{\prime}}-T_{\text {mean }}$ versus $[\mathrm{Fe} / \mathrm{H}]$ (only temperatures obtained from more than one filter are displayed). The dotted lines show the region $|\Delta T| \leq 60 \mathrm{~K}$. The vertical accumulations correspond to the temperatures of the stars of the globular clusters contained in our sample 
Table 7. Temperatures derived for the globular cluster stars of the sample. Column 1 Globular cluster. Column 2: Identification: M 3 nomenclature from Cohen et al. (1978) and Arribas \& Martínez-Roger (1987); M 13, M 92 and M 67 nomenclature from Cohen et al. (1978); M 71 nomenclature from Frogel et al. (1979); 47 Tuc nomenclature from Frogel et al. (1981); NGC 288, NGC 1261, NGC 362 nomenclature from Frogel et al. (1983). Column 3: Metallicity. Column 4: Surface gravity. Column 5: Bolometric flux in $10^{-2} \mathrm{erg} \mathrm{cm}^{-2} \mathrm{~s}^{-1}$. Column 6: Interstellar reddening. Column 7: Temperature derived in band $J$ (units are $\mathrm{K})$. Column 8: Error in $T_{J}$ computed considering errors in $F_{\mathrm{Bol}}$, monochromatic fluxes, $\log (g)$ and $[\mathrm{Fe} / \mathrm{H}]$. Columns 9-10: The same as in Cols. 7-8 for temperature derived in band $H$. Columns 11-12: The same as in Cols. 7-8 for temperature derived in band $K$. Column 13: The weighted mean temperature derived from $T_{J}, T_{H}$ and $T_{K}$. Column 14: Mean error computed by considering linear transmission of errors from Cols. 8, 10 and 12. Column 14. Number of temperatures considered in the average of Col. 13

\begin{tabular}{|c|c|c|c|c|c|c|c|c|c|c|c|c|}
\hline GC & $\overline{I D}$ & {$[\mathrm{Fe} / \mathrm{H}]$} & $\log (g)$ & $F_{\mathrm{Bol}}$ & $\overline{E(B-V)}$ & $\overline{T J}$ & $\Delta T_{J}$ & $\overline{T_{H}}$ & $\Delta T_{H} \quad T_{K}$ & $\Delta T_{K}$ & $T_{\text {mean }}$ & $\Delta T_{\text {mean }} n$ \\
\hline M3 & I21 & -1.50 & 2.50 & $2.982 \mathrm{E}-12$ & 0.00 & 4017 & 207 & 4024 & 844137 & 48 & 4096 & 61 \\
\hline M3 & II18 & -1.50 & 3.50 & $8.147 \mathrm{E}-13$ & 0.00 & 4800 & 118 & 4689 & 854699 & 58 & 4695 & 69 \\
\hline M3 & II 46 & -1.50 & 2.50 & $976 \mathrm{E}-12$ & 0.00 & 3607 & 194 & $<3500$ & -3927 & 52 & 3927 & 52 \\
\hline M3 & III28 & -1.50 & 2.50 & $3.947 \mathrm{E}-12$ & 0.00 & 3843 & 240 & 3935 & 2924073 & 43 & 4073 & 431 \\
\hline M3 & III77 & -1.50 & 2.75 & $2.215 \mathrm{E}-12$ & 0.00 & 4269 & 151 & 4183 & 914224 & 44 & 4211 & 59 \\
\hline M3 & IV25 & -1.50 & 3.00 & $1.561 \mathrm{E}-12$ & 0.00 & 4404 & 144 & 4327 & 724323 & 41 & 4324 & 52 \\
\hline M3 & 193 & -1.50 & 3.50 & $4.270 \mathrm{E}-13$ & 0.00 & 4749 & 128 & 4611 & 784678 & 57 & 4650 & 66 \\
\hline M3 & 216 & -1.50 & 3.25 & $9.340 \mathrm{E}-13$ & 0.00 & 4519 & 139 & 4452 & 854510 & 46 & 4490 & 60 \\
\hline M3 & 1397 & -1.50 & 2.50 & $5.368 \mathrm{E}-12$ & 0.00 & 3810 & 231 & $<3500$ & -3916 & 53 & 3916 & 53 \\
\hline M3 & AA & -1.50 & 2.50 & $4.993 \mathrm{E}-12$ & 0.00 & 3966 & 208 & 3766 & 3883953 & 50 & 3953 & 501 \\
\hline M3 & BI & -1.50 & 3.00 & $872 \mathrm{E}$ & .00 & 4422 & 150 & 4425 & 814487 & 50 & 4463 & 62 \\
\hline M3 & 26 & -1.50 & 2.50 & $203 \mathrm{E}$ & 00 & 3500 & 180 & $<3500$ & -3877 & 7 & 3877 & 57 \\
\hline M3 & 33 & -1.50 & 2.50 & $2.960 \mathrm{E}-12$ & 0.00 & 3620 & 213 & 4057 & 724124 & 37 & 4101 & 49 \\
\hline M3 & 46 & -1.50 & 2.75 & $9.325 \mathrm{E}-13$ & 0.00 & 4416 & 148 & 4533 & 684408 & 47 & 4459 & 56 \\
\hline M3 & 53 & -1.50 & 2.75 & $1.413 \mathrm{E}-12$ & 00 & 3641 & 213 & 4109 & 894392 & 15 & 4297 & 60 \\
\hline M3 & 68 & -1.50 & 2.75 & $867 \mathrm{E}$ & 00 & 3787 & 240 & 4391 & 764334 & 41 & 4354 & 53 \\
\hline M3 & 72 & -1.50 & 2.75 & 2 & 00 & 3845 & 239 & 4124 & 904347 & 2 & 4276 & 57 \\
\hline M3 & 155 & -1.50 & 3.00 & 694 & 00 & 4570 & 133 & 4519 & 744579 & 49 & 4555 & 59 \\
\hline M3 & 311 & -1.50 & 2.50 & 3.10 & 00 & 4057 & 197 & 4153 & 824233 & 42 & 4206 & 56 \\
\hline M3 & 313 & -1.50 & 3.00 & 16 & .00 & 4374 & 153 & 4472 & 804538 & 46 & 4514 & 58 \\
\hline M3 & 428 & 1.50 & 2.50 & $664 \mathrm{E}-12$ & 00 & 41 & 203 & 997 & 239 & 41 & 4239 & 41 \\
\hline M3 & 444 & -1.50 & 2.50 & 208 & 00 & 4189 & 166 & 4093 & 764203 & 13 & 4163 & 55 \\
\hline M3 & 464 & -1.50 & 2.50 & 1.966 & 00 & 3834 & 245 & 4082 & 754118 & 37 & 4106 & 50 \\
\hline M3 & 496 & -1.50 & 2.50 & 37 & 00 & 3713 & 234 & 4044 & 744216 & 3 & 4153 & 54 \\
\hline M3 & 525 & -1.50 & 2.50 & 12 & 00 & 3581 & 180 & 3937 & 853959 & 49 & 3959 & 49 \\
\hline M3 & 557 & -1.50 & 2.50 & 2.45 & 00 & 3783 & 250 & 3902 & 764162 & 10 & 4162 & 40 \\
\hline M3 & 567 & -1.50 & 2.75 & 2 & 00 & 4096 & 183 & 418 & 934322 & 11 & 42 & 57 \\
\hline M3 & 586 & -1.50 & 2.50 & & & 3946 & 209 & $<3500$ & -3911 & 3 & & 53 \\
\hline M3 & 605 & -1.50 & 2.75 & 90 & 00 & 4282 & 157 & 4299 & 694409 & 46 & 4365 & 55 \\
\hline M3 & 617 & -1.50 & 2.50 & 3.962 & 0.00 & 3824 & 242 & 3957 & 1604080 & 44 & 4080 & 44 \\
\hline M3 & 6 & U & 00 & & & & 117 & & 78 & 58 & & 67 \\
\hline M3 & & - & 2.75 & & & & 145 & & 714 & 1 & & 52 \\
\hline M3 & 659 & 1.50 & 2.75 & & & 5 & 245 & 43 & 734 & 5 & 43 & 56 \\
\hline $\mathrm{M}$ & 675 & -1.50 & 2.50 & 2 & & 46 & 222 & & 784169 & 40 & 41 & 53 \\
\hline & I2 & 150 & 3.00 & & & & 120 & & 734 & 1 & & 60 \\
\hline & I18 & -1.50 & 3.00 & 1 . & & 3 & 135 & 46 & 784615 & 51 & 4623 & 62 \\
\hline & I 23 & -1 & 3.00 & & & & 148 & & 714 & 5 & & 55 \\
\hline & I 24 & -1.5 & 2.75 & & & & 149 & & 764 & 5 & & 57 \\
\hline & I 48 & -1.50 & 2.00 & & 0. & $<3500$ & - & 3771 & 643940 & 44 & 3940 & 44 \\
\hline & II 67 & -1.50 & 2.00 & 1 & 0.020 & $<3500$ & & 3786 & 2423903 & 43 & 3903 & 43 \\
\hline & II $^{\prime}$ & -1.50 & 2.75 & & & & 3 & & & 42 & & 57 \\
\hline & II & -1.50 & 2.00 & & & & & & 1983 & 4 & & \\
\hline & III & -1.50 & 2.75 & & & & 165 & & 684277 & 38 & 42 & 49 \\
\hline & III & -1.50 & 2.50 & & & & 204 & 39 & 2874 & 41 & & 41 \\
\hline & III & 1) & 2.50 & & & & & & 76 & & & 49 \\
\hline & III73 & -1.50 & 2.75 & & & & 213 & 41 & 894195 & 44 & 4177 & 59 \\
\hline & IV25 & -1.50 & 3.50 & & & & & & 33 & 114 & & 114 \\
\hline & 84 & -0 . & 2.50 & & & & & & 724 & 53 & & 61 \\
\hline & 94 & -0.08 & 3.25 & & & & 147 & & 1066139 & 103 & 6146 & 116 \\
\hline $\mathrm{M}$ & 105 & -0.08 & 2.00 & $3.534 \mathrm{E}-11$ & 0.06 & 4620 & 146 & 4450 & 774453 & 48 & 4452 & 59 \\
\hline & 10 & & 2.00 & & & & & & & & & \\
\hline & 115 & & 3.25 & & & & & 59 & 015993 & & 60 & 109 \\
\hline & 117 & - & 3.00 & 3 & & & 139 & 5262 & 835208 & 75 & 52 & 92 \\
\hline & 141 & & 2.50 & & 0. & & 127 & 47 & 71 & 53 & 4755 & 61 \\
\hline M6 & 151 & -0.08 & 2.50 & $2.503 \mathrm{E}$ & 0.06 & 4878 & 135 & 4815 & 714792 & 51 & 4802 & 59 \\
\hline
\end{tabular}


Table 7. continued

\begin{tabular}{|c|c|c|c|c|c|c|c|c|c|c|c|c|}
\hline GC & ID & {$[\mathrm{Fe} / \mathrm{H}]$} & $\overline{\log (g)}$ & $F_{\mathrm{Bol}}$ & $E(B-V)$ & $\bar{T} T_{J}$ & $\Delta T_{J}$ & $T_{H}$ & $\Delta T_{H} T_{K}$ & $\Delta T_{K}$ & $T_{\text {mean }}$ & $\Delta T_{\text {mean }} n$ \\
\hline M67 & 164 & -0.08 & 2.50 & $2.474 \mathrm{E}-11$ & 0.06 & 4716 & 149 & 4706 & 774693 & 55 & 4698 & 642 \\
\hline M67 & 170 & -0.08 & 2.00 & $7.033 \mathrm{E}-11$ & 0.06 & 4271 & 177 & 4249 & 724272 & 36 & 4264 & 48 \\
\hline M67 & 193 & 0.08 & 2.75 & $4.670 \mathrm{E}-12$ & 0.06 & 4997 & 133 & 4917 & 804918 & 61 & 4918 & 69 \\
\hline M67 & 223 & 0.08 & 2.50 & $2.407 \mathrm{E}-11$ & 0.06 & 4809 & 128 & 4748 & 734693 & 55 & 4717 & 63 \\
\hline M67 & 224 & -0.08 & 2.75 & $2.031 \mathrm{E}-11$ & 0.06 & 4689 & 153 & 4701 & 774704 & 56 & 4703 & 652 \\
\hline M67 & 231 & -0.08 & 2.75 & $9.721 \mathrm{E}-12$ & 0.06 & 5014 & 132 & 4841 & 724830 & 54 & 4869 & (2) \\
\hline M67 & 244 & 0.08 & 2.75 & $1.752 \mathrm{E}-11$ & 0.06 & 5149 & 140 & 5103 & 795045 & 62 & 5086 & 83 \\
\hline M67 & I17 & 0.08 & 3.00 & $4.120 \mathrm{E}-12$ & 0.06 & 4899 & 144 & 4963 & 794909 & 61 & 4933 & 69 \\
\hline M67 & I 22 & -0.08 & 3.00 & $2.411 \mathrm{E}-12$ & 0.06 & 4996 & 145 & 5060 & 775059 & 64 & 5059 & 70 \\
\hline M67 & III34 & -0.08 & 2.75 & $1.223 \mathrm{E}-11$ & 0.06 & 4577 & 148 & 4660 & 764683 & 54 & 4673 & 03 \\
\hline M67 & IV20 & -0.08 & 2.75 & $1.387 \mathrm{E}-11$ & 0.06 & 4567 & 149 & 4619 & 734632 & 50 & 4627 & 59 \\
\hline M67 & IV77 & -0.08 & 3.00 & $2.551 \mathrm{E}-12$ & 0.06 & 4967 & 144 & 4998 & 784999 & 62 & 4999 & 69 \\
\hline M67 & IV81 & 0.08 & 3.00 & $2.554 \mathrm{E}-12$ & 0.06 & 5373 & 138 & 5385 & 895370 & 78 & 5376 & 96 \\
\hline M71 & B & -0.94 & 1.50 & $3.762 \mathrm{E}-11$ & 0.25 & $<3500$ & - & $<3500$ & -3589 & 72 & 3589 & 72 \\
\hline M71 & A4 & -0.94 & 1.75 & $1.589 \mathrm{E}-11$ & 0.25 & $<3500$ & - & 3780 & 733915 & 68 & 3915 & 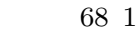 \\
\hline M71 & A6 & -0.94 & 1.75 & $1.419 \mathrm{E}-11$ & 0.25 & 3563 & 212 & 3778 & 723870 & 72 & 3870 & 721 \\
\hline M71 & $\mathrm{S}$ & -0.94 & 2.00 & $6.328 \mathrm{E}-12$ & 0.25 & 4010 & 213 & 4063 & 664139 & 36 & 4112 & \\
\hline M71 & A9 & 0.94 & 2.00 & $6.700 \mathrm{E}-12$ & 0.25 & 3577 & 228 & 3906 & 804008 & 58 & 4008 & 58 \\
\hline M71 & $\mathrm{N}$ & -0.94 & 2.50 & $2.798 \mathrm{E}-12$ & 0.25 & 4773 & 135 & 4787 & 724796 & 52 & 4792 & 60 \\
\hline M71 & A7 & 0.94 & 2.00 & $2.984 \mathrm{E}-12$ & 0.25 & 4106 & 199 & 4360 & 714376 & 42 & 4370 & 4 \\
\hline M71 & A5 & 0.94 & 2.50 & 2.285 & 0.25 & 4310 & 160 & 4494 & 744488 & 47 & 4490 & 572 \\
\hline M71 & $\mathrm{X}$ & -0.94 & 3.00 & $1.004 \mathrm{E}-12$ & 0.25 & 4997 & 145 & 5062 & 825120 & 61 & 5095 & 2 \\
\hline M71 & A3 & 0.94 & 3.00 & 9.522 & 0.25 & 5057 & 140 & 5119 & 65109 & 32 & 5102 & 50 \\
\hline M71 & $\mathrm{C}$ & -0.94 & 2.50 & 1.04 & 0.25 & 4858 & 125 & 4821 & 724797 & 52 & 4807 & 60 \\
\hline M71 & A2 & -0.94 & 2.50 & $9.553 \mathrm{E}-13$ & 0.25 & 4648 & 143 & 4651 & 754657 & 53 & 4655 & 62 \\
\hline M71 & 18 & 94 & 2.50 & & 25 & 4687 & 144 & 4762 & & 53 & & \\
\hline M71 & 19 & 0.94 & 3.00 & 9.73 & 0.25 & 5181 & 142 & 5220 & 95255 & 74 & 5226 & 94 \\
\hline M71 & 21 & 0.94 & 2.00 & 5.14 & 0.25 & 4269 & 166 & 4296 & 44320 & 39 & 43 & 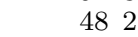 \\
\hline M71 & 29 & 0.94 & 1.50 & 4.89 & 0.25 & 3573 & 198 & 3504 & 03574 & 40 & & 401 \\
\hline M71 & 30 & 0.94 & 1.75 & 1.93 & 0.25 & 3805 & 264 & 3795 & 93897 & 56 & 3897 & 56 \\
\hline M71 & 45 & 0.94 & 1.75 & 1.44 & r & 3706 & 259 & 3770 & & 54 & & \\
\hline M71 & 46 & 0.94 & 1.75 & $1.5 \varepsilon$ & 0.25 & 3588 & 226 & 3747 & 63874 & 54 & 38 & 541 \\
\hline M71 & 75 & 0.94 & 2.75 & -13 & 0.25 & 4920 & 131 & 4740 & 774816 & 54 & 47 & 63 \\
\hline M71 & 76 & 0.94 & 2.50 & 1.56 & 0.25 & 4567 & 136 & 4590 & 714589 & 48 & & \\
\hline M71 & 77 & 0.94 & 1.75 & $1.082 \mathrm{E}-11$ & 0.25 & 3693 & 257 & 3788 & 713906 & 56 & 3906 & ( \\
\hline M71 & 78 & 0.94 & 00 & & 25 & & 161 & 4291 & 654300 & 38 & & 40 \\
\hline M71 & 79 & 0.94 & 2.50 & 12 & 25 & 4353 & 154 & 4475 & 764533 & 44 & 45 & 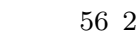 \\
\hline M71 & 113 & 4 & 1.75 & & & $<3500$ & - & 3632 & 1093 & 36 & & 1 \\
\hline M92 & III1: & 2.30 & 2.50 & 12 & 22 & 3945 & 201 & 4033 & 004146 & 0 & 41 & 80 \\
\hline M92 & VII18 & -2.30 & 2.50 & & 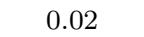 & 41 & 162 & 4174 & 124222 & 50 & & \\
\hline M92 & $\mathrm{X} 49$ & 2.30 & 2.50 & & & & 140 & 4216 & 1054279 & 41 & & 2 \\
\hline M92 & III65 & -2.30 & 2.75 & & 02 & 44 & 142 & 4309 & 864366 & 49 & 43 & - \\
\hline M92 & XII8 & -2.30 & 2.75 & & & & 144 & & 4442 & 54 & & \\
\hline M92 & XI19 & -2.30 & 2.75 & & & 44 & 140 & 4368 & 4433 & 54 & & \\
\hline M92 & II70 & -2.30 & 2.75 & $2.511 \mathrm{E}-12$ & 0.02 & 4569 & 122 & 4522 & 824537 & 48 & 4531 & \\
\hline M92 & & 2.30 & & & & & & & & 51 & & \\
\hline M92 & IV10 & -2.30 & 3.00 & & & & 77 & rer & 4567 & 1 & & \\
\hline M92 & IV2 & -2.30 & 3.00 & & & & & 4576 & 24 & 4 & & \\
\hline M92 & IV114 & -2.30 & 3.50 & & & 46 & 133 & 46 & 14678 & 2 & 46 & \\
\hline & III4 & 2.30 & 3.50 & & & & 131 & 49 & & 5 & & \\
\hline & II 1 & 。 & 3. & & & & & & & 4 & & \\
\hline & 1406 & -0.90 & 2.75 & & & 4 & 152 & 4389 & 4399 & 14 & 43 & 552 \\
\hline & 1407 & & 2.75 & & & & 151 & 45 & 50 & 5 & & \\
\hline & 1414 & -0 & 3.00 & & & 48 & 131 & 48 & 44 & 56 & & \\
\hline 47Tuc & 1421 & -0.90 & 1.50 & $3.504 \mathrm{E}-11$ & 0.04 & $<3500$ & & $<3500$ & -3556 & 60 & 3556 & 100 \\
\hline & 14 & 0.90 & 2.75 & & & & 134 & 4620 & 724 & 49 & & 5 \\
\hline & 15 & & 1.75 & & & & 247 & 38 & & 88 & & \\
\hline & 15 & - & 2.00 & & & & 210 & 39 & 4 & 19 & & \\
\hline & 1513 & -0.90 & 2.00 & $5.731 \mathrm{E}-12$ & & 4221 & 173 & 4163 & 774204 & 41 & 4190 & 542 \\
\hline & 15 & & 2.75 & & & & 157 & 44 & & 4 & & \\
\hline & 16 & & 2.75 & & & & 138 & 46 & 24 & 9 & & \\
\hline & 1603 & 90 & 1.50 & 1.5 & & 37 & 261 & 367 & 83799 & 42 & 37 & \\
\hline & 16 & & 2.50 & & & & 158 & 4216 & 774 & 39 & 4237 & \\
\hline 47 & 2416 & -0.90 & 2.00 & $4.959 \mathrm{E}-12$ & 0.0 & 4039 & 204 & 4040 & 664104 & 53 & 4075 & 592 \\
\hline 47Tuc & 2426 & -0.90 & 1.75 & $9.992 \mathrm{E}-12$ & 0.04 & 3747 & 267 & 3855 & 733940 & 40 & 3940 & \\
\hline
\end{tabular}


Table 7. continued

\begin{tabular}{|c|c|c|c|c|c|c|c|c|c|c|c|c|}
\hline GC & ID & {$[\mathrm{Fe} / \mathrm{H}] 1$} & $\log (g)$ & $F_{\mathrm{Bol}}$ & $E(B-V)$ & $T_{J}$ & $\Delta T_{J}$ & $T_{H}$ & $\Delta T_{H} T_{K}$ & $\Delta T_{K}$ & $T_{\text {mean }}$ & $\Delta T_{\text {mean }} n$ \\
\hline 47Tuc & 2525 & -0.90 & 2.00 & $5.568 \mathrm{E}-12$ & 0.04 & 4215 & 174 & 4181 & 784217 & 41 & 4205 & 542 \\
\hline $47 \mathrm{Tuc}$ & 2603 & -0.90 & 2.50 & $3.630 \mathrm{E}-12$ & 0.04 & 4165 & 174 & 4170 & 774210 & 41 & 4196 & 54 \\
\hline $47 \mathrm{Tuc}$ & 2605 & -0.90 & 2.00 & $5.275 \mathrm{E}-12$ & 0.04 & 4304 & 149 & 4250 & 714204 & 41 & 4221 & 52 \\
\hline 47Tuc & 3407 & 0.90 & 2.50 & $3.187 \mathrm{E}-12$ & 0.04 & 4098 & 190 & 4225 & 774307 & 38 & 4280 & 51 \\
\hline $47 \mathrm{Tuc}$ & 3410 & -0.90 & 2.50 & $2.492 \mathrm{E}-12$ & 0.04 & 4374 & 152 & 4354 & 714368 & 42 & 4363 & 53 \\
\hline 47Tuc & 3501 & -0.90 & 1.75 & $9.431 \mathrm{E}-12$ & 0.04 & 3790 & 268 & 3892 & 773967 & 32 & 3967 & \\
\hline $47 \mathrm{Tuc}$ & 3512 & -0.90 & 1.50 & $2.207 \mathrm{E}-11$ & 0.04 & $<3500$ & - & 3504 & 423671 & 42 & 3671 & 42 \\
\hline 47Tuc & 4411 & -0.90 & 2.75 & $9.268 \mathrm{E}-13$ & 0.04 & 4570 & 137 & 4578 & 704624 & 51 & 4605 & 59 \\
\hline 47Tuc & 4415 & 0.90 & 2.50 & $4.538 \mathrm{E}-12$ & 0.04 & 4620 & 143 & 4692 & 794696 & 57 & 4694 & 66 \\
\hline 47Tuc & 4417 & -0.90 & 3.00 & $7.780 \mathrm{E}-13$ & 0.04 & 4914 & 142 & 4965 & 834983 & 66 & 4975 & 74 \\
\hline 47Tuc & 4418 & 0.90 & 1.75 & $9.454 \mathrm{E}-12$ & 0.04 & 3649 & 246 & 3835 & 713940 & 50 & 3940 & \\
\hline 47Tuc & 4503 & 0.90 & 2.00 & $5.361 \mathrm{E}-12$ & 0.04 & 4255 & 170 & 4289 & 654314 & 39 & 4305 & 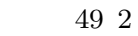 \\
\hline 47Tuc & 4603 & 0.90 & 2.00 & $8.541 \mathrm{~F}$ & 0.04 & 3788 & 269 & 3986 & 764052 & 48 & 4052 & 48 \\
\hline $47 \mathrm{Tuc}$ & 5309 & -0.90 & 1.75 & $8.685 \mathrm{E}-12$ & 0.04 & 3621 & 241 & 3870 & 753983 & 52 & 3983 & 52 \\
\hline 47Tuc & 5312 & -0.90 & 1.75 & $9.396 \mathrm{E}-12$ & 0.04 & 3679 & 254 & 3821 & 693930 & 50 & 3930 & \\
\hline 47Tuc & 5404 & -0.90 & 2.50 & $3.186 \mathrm{I}$ & 0.04 & 4480 & 151 & 4515 & 704533 & 44 & 4526 & \\
\hline $47 \mathrm{Tuc}$ & 5406 & -0.90 & 2.50 & $3.944 \mathrm{E}-12$ & 0.04 & 4165 & 174 & 4130 & 744210 & 41 & 4181 & \\
\hline $47 \mathrm{Tuc}$ & 5422 & 0.90 & 2.00 & 6.072 & 04 & 3915 & 228 & 4040 & 654076 & 31 & 4064 & \\
\hline $47 \mathrm{Tuc}$ & 5427 & -0.90 & 2.50 & $3.460 \mathrm{E}-12$ & 0.04 & 4326 & 141 & 4207 & 784240 & 40 & 4229 & 53 \\
\hline 47Tuc & 5527 & -0.90 & 2.75 & $1.573 \mathrm{E}-12$ & 0.04 & 4406 & 157 & 4488 & 754498 & 46 & 4494 & 57 , \\
\hline 47Tuc & 529 & 0.90 & 1.50 & 1 & 0.04 & $<3500$ & - & 3631 & 92 & 42 & 3792 & 42 \\
\hline $47 \mathrm{Tuc}$ & 5627 & 0.90 & 2.00 & $5.423 \mathrm{l}$ & $0 . U^{2}$ & 4019 & 213 & 4122 & 724204 & 41 & 4174 & 52 \\
\hline $47 \mathrm{Tuc}$ & 5739 & -0.90 & 2.00 & 6.586 & 0.04 & 3903 & 232 & 3980 & 774062 & 48 & 406 & 48 \\
\hline $47 \mathrm{Tuc}$ & 6407 & -0.90 & 2.50 & 3.4391 & & 4409 & 154 & 4352 & 714413 & 46 & 4389 & 56 \\
\hline 47Tuc & 6408 & 0.90 & 2.50 & 3.84 & & 4120 & 181 & 4152 & 764247 & 39 & 421 & \\
\hline 47Tuc & 6502 & -0.90 & 3.00 & 8155 & & 5063 & 136 & 5159 & 5162 & 64 & 51 & \\
\hline $47 \mathrm{Tuc}$ & 6509 & 0.90 & 2.75 & 1.531 & 0. & 4367 & 157 & 4446 & 794527 & 44 & 449 & 01 \\
\hline $47 \mathrm{Tuc}$ & 7320 & -0.90 & 1.50 & $2.087 \mathrm{E}-11$ & 0.04 & $<3500$ & - & $<3500$ & -3615 & 54 & 3615 & \\
\hline 47Tuc & 7502 & -0.90 & 2.75 & $1.120 \mathrm{E}-12$ & 0.04 & 4530 & 142 & 4572 & 684542 & 45 & 4554 & 54 \\
\hline 47Tuc & 7507 & 0.90 & 2.75 & & & $4 \varepsilon$ & 126 & 4702 & 59 & 54 & & \\
\hline 47Tuc & 406 & 0.90 & 2.00 & 2 & & 3856 & 246 & 3991 & 754027 & 8 & 4027 & \\
\hline $47 \mathrm{Tuc}$ & 8416 & -0.90 & 2.75 & 2 & & 4557 & 130 & 4470 & 764464 & 49 & 446 & \\
\hline $47 \mathrm{Tuc}$ & 8517 & -0.90 & 2.00 & 550 & 04 & 4069 & 205 & 4204 & 784252 & 47 & 423 & \\
\hline 47Tuc & 8518 & -0.90 & 2.75 & 2 & 02 & 4574 & 129 & 4549 & 664520 & 44 & 45 & \\
\hline c1261 & 3 & -1.25 & 2.00 & 2 & & 3576 & 238 & 3780 & 1793906 & 50 & 390 & \\
\hline c1261 & & -1.25 & 2.00 & & & 4259 & 150 & 3940 & 843926 & 2 & 39 & \\
\hline 1 & 10 & -1.25 & 2.00 & 2 & & 3653 & 264 & 3812 & 53916 & 1 & 391 & \\
\hline & 11 & -1.25 & 2.00 & & & & 178 & 4078 & & 4 & 40 & \\
\hline & 52 & -1.25 & 2.00 & & & & 183 & 4113 & 734139 & 56 & 412 & 00 \\
\hline ngc1261 & 81 & -1.25 & 2.50 & $1.648 \mathrm{E}-12$ & 0.01 & 4430 & 143 & 4213 & 794218 & 43 & 4216 & \\
\hline ngc288 & 19 & 1.37 & 2.75 & & & 4726 & 137 & 47 & 751 & 54 & 47 & \\
\hline ngc288 & 20 & -1.37 & 2.00 & & & & 185 & 10 & 54073 & 52 & 40 & \\
\hline ngc288 & $\mathrm{C} 23$ & 1.3 & 2.75 & & & & 125 & 4776 & 4767 & 3 & 47 & \\
\hline ngc288 & C32 & -1.37 & 2.75 & & & 4912 & 130 & 4827 & 54829 & 5 & 48 & \\
\hline ngc288 & & 1.3 & 2.50 & & & & & & 774445 & 9 & & \\
\hline 288 & C36 & 1.3 & 2.50 & & & & 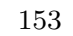 & 43 & 5420 & 8 & 44 & \\
\hline ngc288 & A77 & -1.37 & 2.00 & & & & 139 & 41 & 4208 & 3 & 41 & \\
\hline 288 & A78 & 1 & 2.00 & & & & 156 & & 4101 & 4 & & \\
\hline 288 & A 80 & -1. & 2.50 & & & & 127 & 448 & 774518 & 45 & 450 & ( \\
\hline ngc288 & A96 & -1.37 & 2.00 & $4.967 \mathrm{E}-12$ & 0.02 & 4112 & 175 & 3955 & 884028 & 40 & 4028 & 40 \\
\hline 288 & & & & & & & & & & 7 & & \\
\hline ngc288 & A231 & 1. & 2.50 & & & & & & 04 & 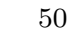 & & \\
\hline & A245 & $1:$ & 2.50 & & & & & 43 & & & 43 & \\
\hline ngc288 & A260 & -1.37 & 1.50 & & & & 150 & 3589 & 1163749 & 4 & 37 & \\
\hline & I & 008 & 2.75 & & & & & & $c e$ & & & \\
\hline 362 & I 23 & 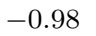 & 2.50 & & & & & 42 & 4294 & 7 & 42 & \\
\hline & $\mathrm{T}$ & -0 & 2.50 & & & & 148 & & 88 & 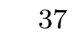 & 42 & \\
\hline & & 0 & & & & & & & 31 & & & \\
\hline 362 & II20 & -0.98 & 2.00 & & & 3644 & 255 & 3881 & 773976 & 53 & 3976 & 00 \\
\hline ngc362 & II40 & -0.98 & 2.75 & $4.568 \mathrm{E}-13$ & 0.04 & 4774 & 128 & 4702 & 794706 & 57 & 4704 & 66 \\
\hline ngc362 & I0 & 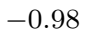 & 2.75 & & & & 10 & & & 0 & & \\
\hline & 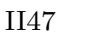 & & 2.75 & & & & $1:$ & & & & & \\
\hline & 11 & & 2.50 & & & & & & 22 & 7 & & \\
\hline & III4 & & 2.50 & & & & 158 & 4331 & 994 & 7 & 43 & \\
\hline ngc362 & III11 & & 2.00 & & & & 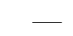 & & 3852 & ) & 38 & \\
\hline ngc36 & $\mathrm{III}_{2}$ & -0. & 2.50 & & & & 150 & & 694369 & 42 & 43 & 02 \\
\hline & 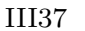 & 0 & 2.50 & & & & 14 & & - & 39 & 43 & \\
\hline ng & [II39 & & 2.00 & & & & 215 & 39 & 3993 & 51 & $39 ؛$ & \\
\hline & III44 & -0.9 & 2.00 & & & 3997 & 211 & 3940 & 814012 & 49 & 4012 & \\
\hline ngc362 & III63 & -0.98 & 2.00 & $6.000 \mathrm{E}-12$ & 0.0 & 3661 & 253 & 3806 & 683905 & 48 & 3905 & \\
\hline
\end{tabular}


Table 7. continued

\begin{tabular}{|c|c|c|c|c|c|c|c|c|c|c|c|c|c|}
\hline$\overline{\mathrm{GC}}$ & ID & {$[\mathrm{Fe} / \mathrm{H}]$} & $\log (g)$ & $F_{\mathrm{Bol}}$ & $E(B-V)$ & $T_{J}$ & $\Delta T_{J}$ & $T_{H}$ & $\Delta T_{H}$ & $T_{K}$ & $\Delta T_{K}$ & $T_{\text {mean }}$ & $\Delta T_{\text {mean }} n$ \\
\hline$\overline{\text { ngc362 }}$ & III70 & -0.98 & 2.00 & $4.3632 \mathrm{E}-12$ & 0.04 & 3902 & 2424 & 4108 & 71 & 4132 & 45 & 4123 & 552 \\
\hline ngc362 & IV84 & -0.98 & 2.00 & $3.896 \mathrm{E}-12$ & 0.04 & $<3500$ & 3 & 3950 & 81 & 4021 & 49 & 4021 & 49 \\
\hline 2 & IV91 & -0.98 & 2.50 & $1.981 \mathrm{~F}$ & 0.04 & 4210 & 175 & 4366 & 72 & 4382 & 44 & 4376 & 55 \\
\hline ngc $36:$ & IV100 & -0.98 & 2.00 & $5.923 \mathrm{I}$ & 0.04 & $<3500$ & -3 & 3702 & 156 & 3856 & 55 & 3856 & 55 \\
\hline ngc362 & $\mathrm{V} 2$ & -0.98 & 1.50 & $7.716 \mathrm{E}-12$ & 0.04 & $<3500$ & -3 & 3777 & $69:$ & 3779 & 50 & 3779 & 50 \\
\hline
\end{tabular}

In order to estimate the error of the mean temperature, a linear transmission of the errors was considered, given that the errors in each band are not totally independent:

$$
\Delta T_{\mathrm{IRFM}}=\frac{N}{\sum_{i=J, H, K, L} \frac{1}{\Delta T_{i}}},
$$

where $N$ is the number of bands considered and the error in the temperature of each band is defined by

$$
\begin{array}{r}
\left(\Delta T_{i}\right)^{2}=\left[\frac{\partial T_{i}}{\partial\left[q\left(\lambda_{i}\right) R\left(\lambda_{i}\right)\right]}\right]^{2}\left(\Delta\left[q\left(\lambda_{i}\right) R\left(\lambda_{i}\right)\right]\right)^{2} \\
+\left[\frac{\partial T_{i}}{\partial[\mathrm{Fe} / \mathrm{H}]}\right]^{2}(\Delta[\mathrm{Fe} / \mathrm{H}])^{2}+\left[\frac{\partial T_{i}}{\partial \log (g)}\right]^{2}(\Delta \log (g))^{2} .
\end{array}
$$

The quantities in square brackets have been estimated by considering finite-difference interpolation with the help of grids of values similar to those displayed in Tables 1-4 with a finer spacing. Over $5000 \mathrm{~K}$, the temperatures in the three (four) bands enter the average with similar weight. In that range, the assignment of weights automatically takes into account the uneven sensitivity of the IRFM in the different bands and the individual quality of IR photometry. However, below $5000 \mathrm{~K}$ only $T_{H}, T_{K}$ and $T_{L^{\prime}}$ have been considered in the average, since $R_{J}$ is a very insensitive indicator of temperature for the cooler stars. Under $4000 \mathrm{~K}$, only $T_{K}$ and $T_{L^{\prime}}$ have been considered. This is due to the fact that the coolest models show in the $H$ band a local maximun of flux which is not observed in IR spectra (Lançon \& Rocca-Volmerange 1992).

The mean error in the final temperatures is around $1-2 \%$. Note however, that the uncertainties in the temperatures derived under $4000 \mathrm{~K}$ are greater than the errors determined from Eq. (8) due to the model imperfections in this range caused by the absence of important sources of opacity associated with certain molecules. Likewise, the IRFM is difficult to apply at temperatures above $8000 \mathrm{~K}$ because, as these stars emit a substantial proportion of energy at short wavelengths, the correction for insterstellar extinction and the determination of the bolometric flux are rather uncertain. For these reasons, the temperatures outside the range $4000 \mathrm{~K}<T_{\text {eff }}<8000 \mathrm{~K}$ have a lower level of accuracy, and the error-bars quoted in Tables 6 and 7 have to be considered, in some cases, as lower estimates.

We show in Figs. 5 and 6 the difference between $T_{J}$, $T_{H}, T_{K}$ and $T_{L^{\prime}}$, and the average temperature adopted.
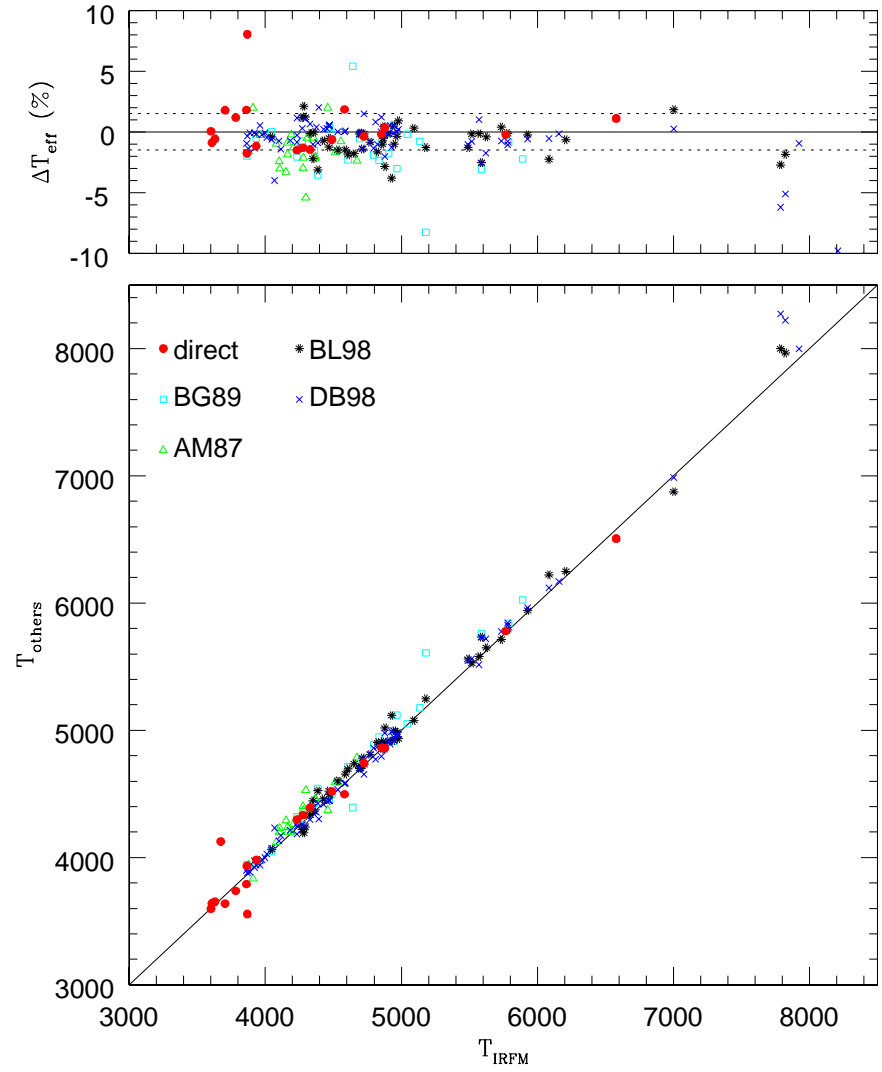

Fig. 7. Differences between the temperatures derived in this work $\left(T_{\text {IRFM }}\right)$ and those derived by other authors. circles: Direct measurements: C76, R80, BR87, H89, M91, and WF87; squares: Bell \& Gustafsson (1989), triangles: Arribas \& Martínez-Roger (1987). stars: Blackwell \& Lynas-Gray (1998). In the upper figure the lines corresponding to the mean internal error of the work $( \pm 1.5 \%)$ are shown

The individual residuals reveal that the dispersion is compatible with the estimated errors derived from the uncertainties in the input parameters of the IRFM. They follow approximately a normal distribution both with temperature and metallicity. As expected, the uncertainties are greater for temperatures obtained from $R_{J}$ factors, due to the lower sensitivity of the IRFM in this band and the greater photometric error in the measurement of magnitude $J$. The consistency of $T_{J}$ and $T_{H}$ is good over $4500 \mathrm{~K}$; however, under this temperature noteworthy discrepancies appear to be due to the fact that $R_{J^{-}}$and $R_{H}$-factors lose their sensitivity to temperature in this range. 

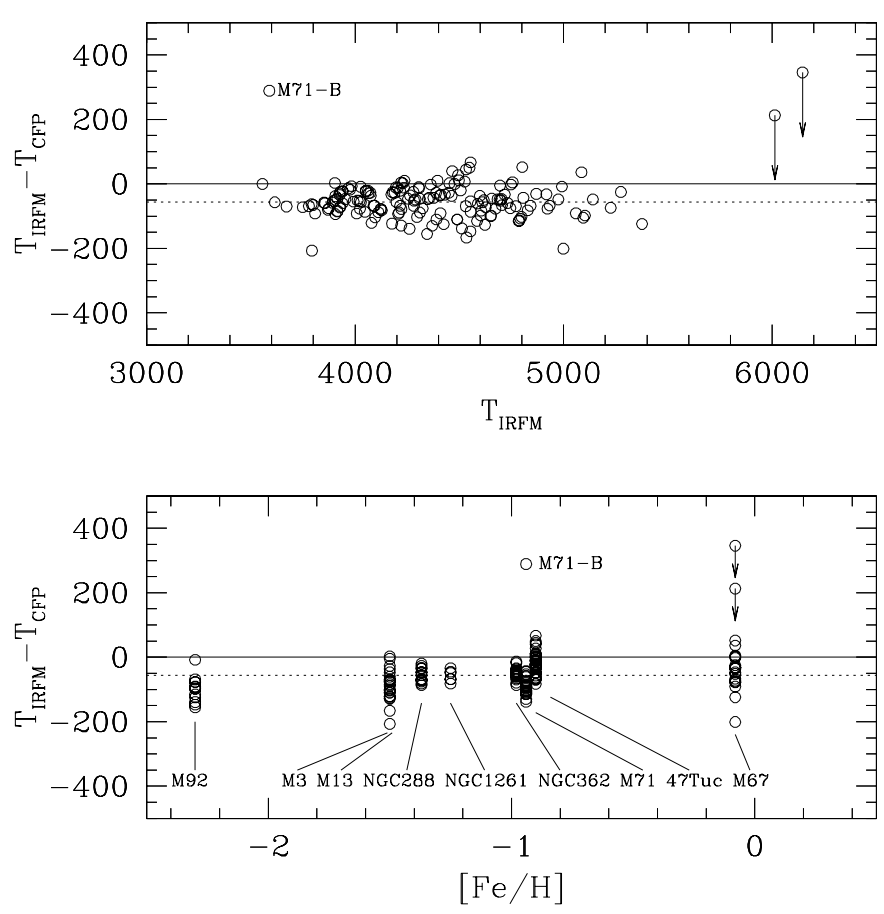

Fig. 8. Differences between the temperatures derived in this work $\left(T_{\text {IRFM }}\right)$ and those derived by Frogel et al. (1979, 1981, $1983)$ and Cohen et al. (1978) ( $\left.T_{\mathrm{CFP}}\right)$. Top: Differences against $T_{\text {IRFM }}$. Bottom: Differences against metallicity. The differences are consistent with a zero-point offset amounting to $56 \mathrm{~K}$ (dotted line)

Table 8. Comparison between the temperatures derived in the present work (Col. 3) and those derived by direct methods (Col. 2). When several direct measurements were available we have considered the average value. The temperatures of the Sun and Procyon, measured in Paper I, are also listed. The mean difference $T_{\text {direct }}-T_{\text {IRFM }}$ is $3 \pm 51 \mathrm{~K}$

\begin{tabular}{lccr}
\hline \multicolumn{1}{c}{ Star } & $T_{\text {direct }}(\mathrm{K})$ & $T_{\text {IRFM }}(\mathrm{K}) \Delta T(\mathrm{~K})(\%)$ \\
\hline HR 7635 & 3556 & 3867 & -8.7 \\
HR 8775 & 3598 & 3600 & -0.1 \\
HR 911 & 3638 & 3704 & -1.8 \\
HR 4902 & 3639 & 3607 & +0.9 \\
HR 2286 & 3652 & 3631 & +0.6 \\
HR 337 & 3738 & 3783 & -1.2 \\
HR 3705 & 3791 & 3861 & -1.8 \\
HR 1457 & 3934 & 3866 & +1.7 \\
HR 6705 & 3980 & 3934 & +1.2 \\
HR 5301 & 4125 & 3672 & +10.1 \\
HR 5340 & 4297 & 4233 & +1.5 \\
HR 603 & 4333 & 4277 & +1.3 \\
HR 165 & 4392 & 4329 & +1.4 \\
HR 168 & 4497 & 4582 & -1.9 \\
HR 617 & 4519 & 4490 & +0.6 \\
HR 7949 & 4743 & 4725 & +0.4 \\
HR 2990 & 4863 & 4854 & +0.2 \\
HR 7776 & 4860 & 4878 & -0.4 \\
Sun & 5780 & 5767 & +0.2 \\
HR 2943 & 6506 & 6579 & -1.1 \\
\hline
\end{tabular}

Table 9. Comparison between the temperatures derived in the present work (Col. 2) and those derived by Arribas \& MartínezRoger (1987) (Col. 3). The mean difference $T_{\mathrm{IRFM}}-T_{\mathrm{AM} 89}$ is $-1.3 \pm 1.4 \%$ (without M3-53)

\begin{tabular}{ccccr}
\hline Star & $T_{\text {IRFM }}(\mathrm{K})$ & $T_{\text {AM87 }}(\mathrm{K})$ & $\Delta T(\mathrm{~K})$ & $\Delta T(\mathrm{~K})(\%)$ \\
\hline M3-26 & 3877 & 3940 & -63 & -1.6 \\
M3-33 & 4101 & 4200 & -99 & -2.4 \\
M3-46 & 4459 & 4370 & +89 & +2.0 \\
M3-53 & 4297 & 4530 & -233 & -5.4 \\
M3-68 & 4354 & 4375 & -21 & -0.5 \\
M3-72 & 4276 & 4404 & -128 & -3.0 \\
M3-155 & 4555 & 4590 & -35 & -0.7 \\
M3-311 & 4206 & 4260 & -54 & -1.3 \\
M3-313 & 4514 & 4590 & -76 & -1.7 \\
M3-428 & 4239 & 4300 & -61 & -1.4 \\
M3-444 & 4163 & 4200 & -37 & -0.9 \\
M3-464 & 4106 & 4230 & -124 & -3.0 \\
M3-496 & 4153 & 4290 & -137 & -3.3 \\
M3-525 & 3959 & 3970 & -11 & -0.3 \\
M3-557 & 4162 & 4240 & -78 & -1.9 \\
M3-567 & 4279 & 4370 & -91 & -2.1 \\
M3-586 & 3911 & 3834 & +77 & +2.0 \\
M3-605 & 4365 & 4460 & -95 & -2.2 \\
M3-617 & 4080 & 4120 & -40 & -1.0 \\
M3-627 & 4672 & 4784 & -112 & -2.4 \\
M3-650 & 4306 & 4330 & -24 & -0.6 \\
M3-659 & 4370 & 4460 & -90 & -2.1 \\
M3-675 & 4191 & 4200 & -9 & -0.2 \\
\hline & & & &
\end{tabular}

\section{Comparison with other determinations}

In this section, we provide the comparison of our temperatures with those derived by other authors for common stars in the sample. We show differences found in Figs. 7 and 8. Furthermore, a detailed analysis of the scale of temperatures derived from the present work will be given in a subsequent paper by considering the mean relations $T_{\text {eff }}:[\mathrm{Fe} / \mathrm{H}]$ and the $U B V R I J H K$ and uvby photometric colours.

\section{Direct methods}

There is a group of 18 giant stars in the sample whose diameters have been measured by optical methods (Code et al. (1976; C76), Ridgway et al. (1980; R80), Di Benedetto \& Rabbia (1987; BR87), Hutter et al. (1989; H89), Mozurkewich et al. (1991; M 91), and White \& Feiermann (1987; WF87)). In Table 8, we provide the comparison between IRFM temperatures and those obtained considering their measured angular diameters and bolometric fluxes. If we consider the typical errors of both the direct method and the IRFM the differences observed are within the error-bars. Excluding HR 7635 and HR 5301, the average difference $T_{\text {direct }}-T_{\text {IRFM }}$ 
amounts to $0.06 \pm 1.25 \%$. Moreover, no apparent trend of the differences with temperature is observed.

\section{Arribas \& Martínez-Roger (1987, AM87)}

There are 23 stars of M 3 from the article of Arribas \& Martínez-Roger (1987, AM87), based on the application of the IRFM and using the empirical absolute flux calibration of the IR flux of Vega derived by Mountain et al. (1985), and the models of Kurucz (1979) and Gustaffson et al. (1975), which are common with our work (Table 9). Temperatures derived by AM87 are, on average, $55 \mathrm{~K}$ hotter, with a standard deviation amounting to $59 \mathrm{~K}$. No obvious trend with temperature can be seen in the differences (Fig. 7). The shift in temperatures, amounting to $1.3 \%$, might be explained by taking into account the different absolute flux calibration, and the improvement of Kurucz's models.

\section{Bell $\& 5$ Gustafsson (1989, BG89)}

This work contains 25 stars in common with the present sample. BG89 temperatures are based on the IRFM corrected with IR synthetic colours. The differences listed in Table 10 are compatible with a zero-point shift of $65 \mathrm{~K}$ $\left(T_{B G 89}\right.$ are hotter) or $1.3 \pm 1.1 \%$. These differences are probably connected with the differences in the bolometric fluxes adopted in both works (Sect. 4.2).

\section{Blackwell \& Lynas-Gray (1998; BL98)}

This extensive study provides temperatures for 420 stars (A0-K3) with luminosity classes between II and $\mathrm{V}$, incorporating previous results of similar accuracy (Blackwell et al. 1990 and Blackwell \& Lynas-Gray 1994). It is a good source for comparison, given that it is based on the IRFM with the same models adopted in the present work, the basic differences being the calibration of the IR flux of Vega, and the scale of bolometric fluxes. In Fig. 7, we show the differences in temperature for the 50 Population I giants common to the present sample. On average, $T_{\mathrm{BL} 98}$ are $36 \pm 67 \mathrm{~K}$ hotter than ours; no apparent trend of the differences versus $T_{\text {eff }}$ is seen. This shift in temperature $(0.70 \% \pm 1.25 \%)$ is compatible with the difference in the bolometric fluxes (around 1.5\%) found in Sect. 4.2.

\section{Di Benedetto (1998; DB98)}

This work reports on the implementation of the empirical surface brightness technique using Jonhson $(V-K)$
Table 10. Comparison between the temperatures derived in the present work (Col. 2) and those derived by Bell \& Gustafsson (1989) (Col. 3). The mean difference $T_{\mathrm{IRFM}}-T_{\mathrm{BG} 89}$ is $-65 \pm$ $56 \mathrm{~K}$ discarding HR 4247 and HR 5889

\begin{tabular}{cccrr}
\hline Star & $T_{\text {IRFM }}(\mathrm{K})$ & $T_{\text {BG89 }}(\mathrm{K})$ & $\Delta T(\mathrm{~K}) \Delta T(\mathrm{~K})(\%)$ \\
\hline HR 0617 & 4490 & 4499 & -9 & -0.2 \\
HR 1457 & 3866 & 3943 & -77 & -2.0 \\
HR 2990 & 4854 & 4896 & -42 & -0.9 \\
HR 5340 & 4233 & 4321 & -88 & -2.1 \\
HR 6705 & 3934 & 3955 & -21 & -0.5 \\
HR 0219 & 5789 & 5839 & -49 & -0.8 \\
HR 0434 & 4042 & 4046 & +0 & +0.0 \\
HR 0464 & 4359 & 4425 & -66 & -1.5 \\
HR 1907 & 4693 & 4719 & -26 & -0.6 \\
HR 3323 & 5136 & 5176 & -40 & -0.8 \\
HR 3403 & 4387 & 4542 & -155 & -3.5 \\
HR 4247 & 4643 & 4392 & +251 & +5.4 \\
HR 4932 & 5043 & 5052 & -9 & -0.2 \\
HR 4983 & 5892 & 6024 & -132 & -2.2 \\
HR 5429 & 4271 & 4303 & -32 & -0.7 \\
HR 5889 & 5180 & 5608 & -428 & -8.2 \\
HR 6220 & 4942 & 4913 & +29 & +0.6 \\
HR 6603 & 4533 & 4603 & -70 & -1.5 \\
HR 6770 & 4969 & 5120 & -151 & -3.0 \\
HR 6869 & 4835 & 4949 & -114 & -2.3 \\
HR 7429 & 4473 & 4456 & +17 & +0.4 \\
HR 7615 & 4796 & 4887 & -91 & -1.9 \\
HR 7957 & 4908 & 4996 & -88 & -1.8 \\
HR 8255 & 4609 & 4715 & -106 & -2.2 \\
HR 4883 & 5589 & 5841 & -172 & -3.1 \\
\hline & & & &
\end{tabular}

colours for 537 dwarf and giant stars A-K. There are 70 giant and subgiant stars of DB98 common both to Paper I and the present work. Below $7000 \mathrm{~K}$, the agreement is fairly good. The DB98 temperatures are $12 \mathrm{~K}$ hotter with a dispersion of $42 \mathrm{~K}(0.23 \pm 0.93 \%)$. However, for the four stars with $T_{\text {IRFM }}>7000 \mathrm{~K}$ a difference of 400 $\pm 300 \mathrm{~K}$ is observed (the DB98 temperatures are hotter). This fact is probably related to the difficulty in estimating bolometric fluxes for early-type stars.

Cohen et al. (1978), Frogel et al. (1979, 1981, 1983); CFP

The scale of temperatures defined by CFP has been adopted as standard in many studies devoted to the analysis of chemical abundances of giant stars and the calibration of stellar evolution models of the RGB. In the series of papers above mentioned, the authors provide effective temperatures for an extended sample of Red Giant Branch stars of globular clusters. The effective temperatures derived by CFP are based on atmosphere models and multicolour photometry (a brief description of the method used to derive temperatures is detailed in Frogel et al. 1981). In Fig. 8, we show the differences observed between $T_{\text {IRFM }}$ and $T_{\mathrm{CFP}} . \mathrm{In}$ average, $T_{\mathrm{CFP}}$ are $56 \mathrm{~K}$ hotter than $T_{\mathrm{IRFM}}$ 
with a dispersion of $46 \mathrm{~K}$. No apparent trend of the differences with $T_{\text {eff }}$ or $[\mathrm{Fe} / \mathrm{H}]$ is appreciated.

In summary, temperatures derived here are comparable with those derived by other authors. On the one hand, the differences and the dispersion of the differences found with other works based on the IRFM are within the errorbars of both accidental errors (i.e. uncertainty on the bolometric and monochromatic fluxes and other input parameters of the IRFM) and systematic errors (i.e. uncertainty in the absolute flux calibration as commented in Sect. 4.1 and different grids of model fluxes). On the other hand, the differences and the dispersion of the differences found with direct methods and surface brightness method are consistent with the combination of our internal error estimates and theirs.

\section{Summary}

The IRFM has been applied to a sample of approximately 500 giant stars later than F0, which cover the metallicity range $(0.5,-3.5)$. Near-IR monochromatic fluxes have been used in order to derive $T_{J}, T_{H}, T_{K}$ and $T_{L^{\prime}}$ for each star. The uncertainties in the input parameters needed to apply the IRFM and the induced errors on the three/four temperatures derived have been computed. The consistency of the temperatures derived in the three different bands is fairly good above $4000 \mathrm{~K}$. The final temperature for each star in the sample has been derived considering the mean of $T_{J}, T_{H}, T_{K}$ and $T_{L^{\prime}}$ weighted with the inverse of their errors. From the analysis of the systematic errors associated with the uncertainty in the absolute flux calibration in the near-IR, the expected indeterminacy of the zero point of the temperature scale should be around $1 \%$. However, the good agreement between the IRFM and direct temperatures of a sample of 18 giant stars suggests a lower uncertainty. The internal estimated precision for the final temperatures, considering the effect of accidental errors, is around $1.5 \%$. The comparison with other works also based on semi-empirical and empirical methods shows slight discrepancies which may be explained by considering the internal errors affecting the determination of temperatures.

Acknowledgements. We are grateful to the referee, Dr. LynasGray, for his careful assessment of the paper and for many comments and suggestions which have certainly improved the final version. We are also grateful to Terry Mahoney for correcting this paper for English and style. We have made use for this research of the SIMBAD database, operated at CDS, Strasbourg, France.

\section{References}

Alonso A., Arribas S., Martínez-Roger C., 1994a, A\&A 282, 684 (Paper II)

Alonso A., Arribas S., Martínez-Roger C., 1994b, A\&AS 107, 365

Alonso A., Arribas S., Martínez-Roger C., 1995, A\&A 297, 197 (Paper III)

Alonso A., Arribas S., Martínez-Roger C., 1996a, A\&AS 117, 227 (Paper I)

Alonso A., Arribas S., Martínez-Roger C., 1996b, A\&A 313, 873

Alonso A., Arribas S., Martínez-Roger C., 1998, A\&AS 131, 209 (Paper IV)

Anthony-Twarog B.J., Twarog B.A., 1994, AJ 107, 1577

Arribas S., Caputo F., Martínez-Roger C., 1991, A\&AS 88, 19 (AM87)

Arribas S., Martínez-Roger C., 1987, A\&A 178, 107

Bell R.A., Gustafsson B., 1989, MNRAS 236, 653 (BG89)

Bell R.A., 1992, MNRAS 257, 423

Bessell M.S., Castelli F., Plez B., 1998, A\&A 333, 231 (BCP98)

Blackwell D.E., Petford A.D., Arribas S., Haddock D.J., Selby M.J., 1990, A\&A 232, 396

Blackwell D.E., Petford A.D., 1991, A\&A 250, 459

Blackwell D.E., Lynas-Gray A.E., 1994, A\&A 282, 899

Blackwell D.E., Lynas-Gray A.E., 1998, A\&AS 129, 505 (BL98)

Böhm-Vitense E., 1981, ARA\&A 19, 295

Burstein D., Heiles C., 1982, AJ 87, 1165

Carney B.W., 1979, ApJ 233, 211

Cayrel de Strobel G., Soubiran C., Friel E.D., et al., 1997, A\&AS 124, 299

Clark F.O., 1996, PLEXUS Version 2.1a, CD-ROM. Hanscom AFB, MA: Phillips Lab., Dir. Geophys., Air Force Mater. Command

Code A.D., Davis J., Bless R.C., Hanbury-Brown R., 1976, ApJ 203, 417 (C76)

Cohen J.G., Frogel J.A., Persson S.E., 1978, ApJ 222, 165

Crawford D.L., 1975, AJ 80, 955

Di Benedetto G.P., 1993, A\&A 270, 315

Di Benedetto G.P., 1998, A\&A 339, 858 (DB98)

Di Benedetto G.P., Rabbia Y., 1987, A\&A 188, 114 (BR87)

Dyck H.M, van Belle G.T., Thompson R.R., 1998, AJ 116, 981

Dreiling L.A., Bell R.A., 1980, ApJ 241, 736

Flower P.J., 1996, ApJ 469, 365 (F96)

Frogel J.A., Persson S.E., Cohen J.G., 1979, ApJ 227, 499

Frogel J.A., Persson S.E., Cohen J.G., 1981, ApJ 246, 842

Frogel J.A., Persson S.E., Cohen J.G., 1983, ApJS 53, 713

Gustafsson B., Bell R.A., Eriksson K., Nordlund A., 1975, A\&A 42, 407

Hakkila J., Myers J.M., Stidham B.J., Hartmann D.H., 1997, AJ 114, 2043

Hayes D.S., Latham D.W., 1975, ApJ 197, 593

Hutter D.J., Johnston K.J., Mozurkewich D., et al., 1989, ApJ 340, 1103 (H89)

Lançon A., Rocca-Volmerange B., 1992, A\&AS 96, 593

Landolt-Börnstein, 1982, New Series, Gp VI, Vol. 2, Astronomy and Astrophysics, Subvolume C. Springer, Berlin-Heidelberg-New York

Kurucz R.L., 1993, CD-ROM \# 13

Kurucz R.L., 1995, CD-ROM \# 23

Malagnini M.L., Morossi C., Buser R., Parthasarathy M., 1992, A\&A 267, 558 
Morossi C., Franchini M., Malagnini M.L., et al., 1993, A\&A 277,173

Mozurkewich D., Johnston K.J., Simon R.S., et al., 1991, AJ 101, 2207 (M91)

Peterson C.J., 1986, PASP 98, 192

Petford A.D., Blackwell D.E. Booth A.J., et al., 1988, A\&A 203, 341

Plez, B., Brett J.M., Nordlund Å., 1992, A\&A 256, 551

Richichi A., Di Giacomo A., Lisi F., Calamai G., 1992, A\&A 265,535
Ridgway S.T., Joyce R.R., White N.M., Wing R.F., 1980, ApJ 235, $126(\mathrm{R} 80)$

Selby M.J., Hepburn I., Blackwell D.E., et al., 1988, A\&AS 74, 127

Tüg H., White N.M., Lockwood G.W., 1977, A\&A 61, 67

Walker R.G., Cohen M., 1992, An atlas of selected calibrated stellar spectra. NASA Contractor Report 177604, NASA Ames Research Center, Moffett Field. California 940351000

White N.M., Feierman B.H., 1987, AJ 94, 751 (WF87) 